\title{
Angiopoietin-like protein 1 suppresses SLUG to inhibit cancer cell motility
}

\author{
Tsang-Chih Kuo, ${ }^{1}$ Ching-Ting Tan, ${ }^{2}$ Yi-Wen Chang, ${ }^{3,4}$ Chih-Chen Hong, ${ }^{5,6}$ Wei-Jiunn Lee, ${ }^{7}$ \\ Min-Wei Chen, ${ }^{8}$ Yung-Ming Jeng, ${ }^{9}$ Jean Chiou, ${ }^{6}$ Pei Yu, ${ }^{1}$ Pai-Sheng Chen, ${ }^{10,11}$ \\ Ming-Yang Wang, ${ }^{12}$ Michael Hsiao, ${ }^{4}$ Jen-Liang Su, 5,6,13 and Min-Liang Kuo ${ }^{1,14}$
}

\begin{abstract}
${ }^{1}$ Graduate Institute of Toxicology, College of Medicine, National Taiwan University, Taipei, Taiwan. ${ }^{2}$ Department of Otolaryngology, National Taiwan University Hospital, Taipei, Taiwan. ${ }^{3}$ Graduate Institute of Biochemistry and Molecular Biology, National Yang-Ming University, Taipei, Taiwan. ${ }^{4}$ Genomics Research Center, Academia Sinica, Taipei, Taiwan. ${ }^{5}$ Graduate Institute of Cancer Biology, China Medical University, Taichung, Taiwan. ${ }^{6}$ Center for Molecular Medicine, China Medical University Hospital, Taichung, Taiwan. ${ }^{7}$ Department of Computer Science and Engineering, Yuan Ze University, Chung-Li, Taiwan. ${ }^{8}$ Department of Oncology and ${ }^{9}$ Department of Pathology, National Taiwan University Hospital, Taipei, Taiwan. ${ }^{10}$ Department of Medical Laboratory Science and Biotechnology and ${ }^{11}$ The Institute of Basic Medical Sciences, National Cheng Kung University, Tainan, Taiwan. ${ }^{12}$ Department of Surgery, National Taiwan University Hospital, Taipei, Taiwan. ${ }^{13}$ Department of Biotechnology, Asia University, Taichung, Taiwan. ${ }^{14}$ Graduate Institute of Biochemical Sciences, College of Life Science, National Taiwan University, Taipei, Taiwan.
\end{abstract}

\begin{abstract}
Angiopoietin-like protein 1 (ANGPTL1) is a potent regulator of angiogenesis. Growing evidence suggests that ANGPTL family proteins not only target endothelial cells but also affect tumor cell behavior. In a screen of 102 patients with lung cancer, we found that ANGPTL1 expression was inversely correlated with invasion, lymph node metastasis, and poor clinical outcomes. ANGPTL1 suppressed the migratory, invasive, and metastatic capabilities of lung and breast cancer cell lines in vitro and reduced metastasis in mice injected with cancer cell lines overexpressing ANGPTL1. Ectopic expression of ANGPTL1 suppressed the epithelial-to-mesenchymal transition (EMT) by reducing the expression of the zinc-finger protein SLUG. A microRNA screen revealed that ANGPTL1 suppressed SLUG by inducing expression of miR-630 in an integrin $\alpha_{1} \beta_{1} /$ FAK/ERK/SP1 pathway-dependent manner. These results demonstrate that ANGPTL1 represses lung cancer cell motility by abrogating the expression of the EMT mediator SLUG.
\end{abstract}

\section{Introduction}

Metastasis is the most important contributor to the mortality of patients with cancer (1). The pathogenesis of cancer metastasis involves increased cell invasion, angiogenesis, cell proliferation, loss of cellular adhesion, survival in the circulation, entry into new tissue, and eventual colonization of distant organs (2). Recently, a family of proteins structurally similar to the angiopoietins was identified as angiopoietin-like proteins (ANGPTLs), which comprise 7 proteins, ANGPTL1-ANGPTL7 (3). ANGPTLs do not bind to the angiopoietin receptor Tie 2 or the elated protein Tie1, which indicates that the functional mechanism of ANGPTL proteins may be different from that of angiopoietins (3-8). Several studies show that ANGPTL proteins, similar to angiopoietins, potently regulate angiogenesis. Some ANGPTL proteins also involve multibiological properties, such as cancer cell invasion (4), lipid metabolism $(3,5,6)$, hematopoietic stem cell activity (7), and inflammation (8). ANGPTL1 has been reported as an antiangiogenic protein by inhibiting the proliferation, migration, tube formation, and adhesion of endothelial cells as well as tumor growth $(9,10)$; however, little is known about whether ANGPTL1 can influence the malignant properties of cancer cells and the exhaustive mechanisms in cancer progression.

The concept of epithelial-to-mesenchymal transition (EMT) that initially developed in the field of embryology has been extended to explain cancer progression and metastasis (11). Mesenchymal-to-epithelial transition (MET) is the reverse process of EMT, involving the stabilization of distant metastasis by allowing cancer

Authorship note: Ching-Ting Tan and Yi-Wen Chang contributed equally to this work.

Conflict of interest: The authors have declared that no conflict of interest exists. Citation for this article: J Clin Invest. 2013;123(3):1082-1095. doi:10.1172/JCI64044 cells to regain epithelial properties. One of the hallmarks of EMT in cancer is the downregulation of E-cadherin, which is thought to be a repressor of invasion and metastasis. The SNAI, TWIST, and ZEB families have been found to act as oncogenic transcription factors by suppressing E-cadherin expression (12-15). MicroRNAs (miRNAs) are small, endogenous, 21- to 23-nucleotide noncoding RNAs belonging to a novel class of gene regulators that play critical roles in physiologic and pathologic processes, including development, viral infection, and cancer $(16,17)$. Recent studies demonstrate that miRNAs might affect multiple steps of metastasis, including cancer cell migration, invasion, and intravasation (18). Actually, the miR200 family was shown to regulate EMT by inhibiting ZEB1/2. The understanding of miRNA and EMT mechanisms in tumor metastasis is incomplete and remains to be further investigated.

In this study, we reveal what we believe to be the novel mechanism involved in ANGPTL1-mediated suppression of invasion and metastasis of cancer cells. We demonstrate that ANGPTL1 induces MET by downregulation of SLUG through transcriptional regulation of miR-630. Furthermore, we show that ANGPTL1 interacts with integrin $\alpha_{1} \beta_{1}$ and represses its downstream signaling to reduce cancer cell migration and invasion. These findings suggest that ANGPTL1 inhibits cancer cell motility and invasiveness through the integrin $\alpha_{1} \beta_{1} /$ FAK/ERK/SP1/miR-630/SLUG pathway.

\section{Results}

ANGPTL1 expression inversely correlates with poor prognosis of patients with cancer. To determine the clinical significance of ANGPTL1 expression in patients with cancer, we analyzed samples from a cohort of 102 human patients with lung cancer using immunohistochemical staining with a monoclonal antibody specific against ANGPTL1. The antibody specificity was defined by 
Lung cancer

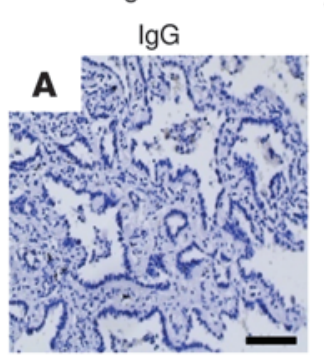

E
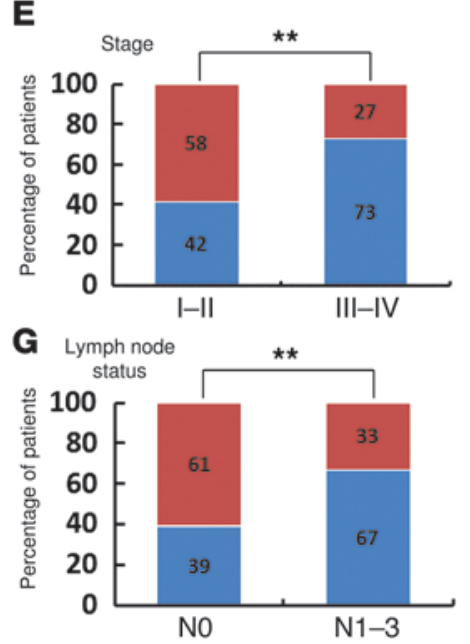

I

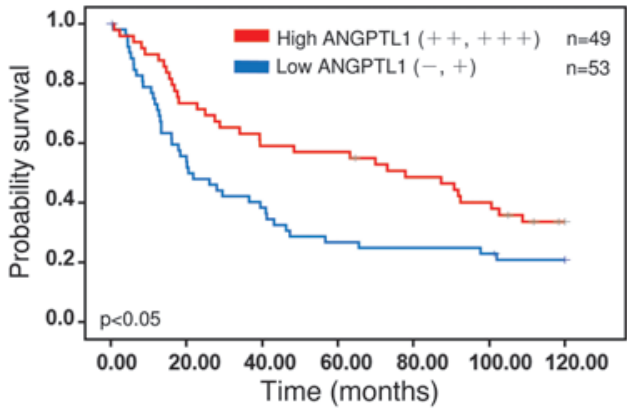

$\mathbf{F}$

H
ANGPTL1
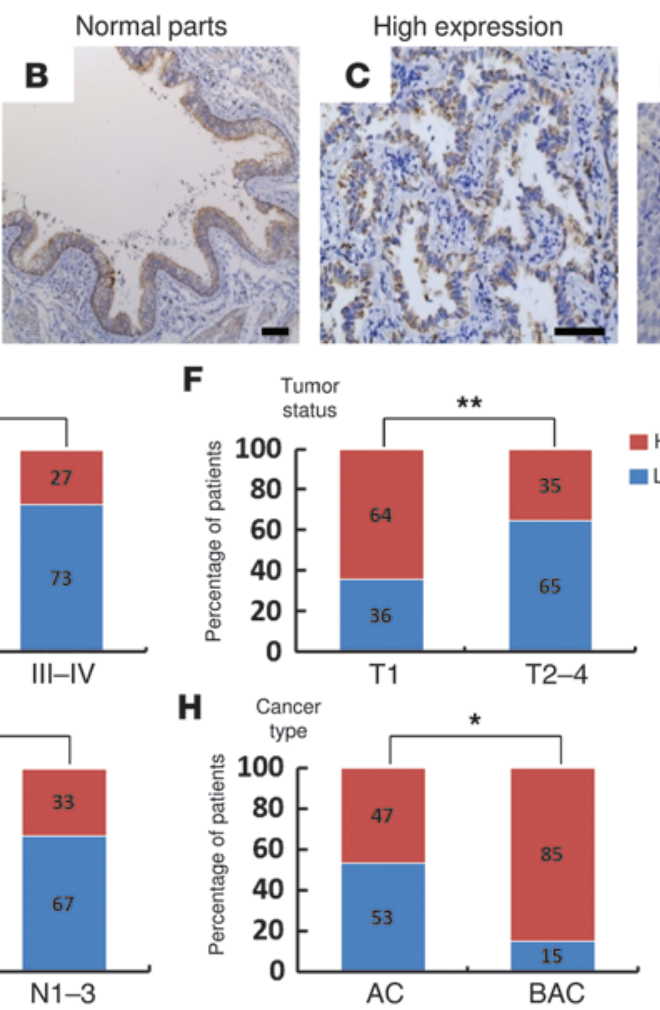

J

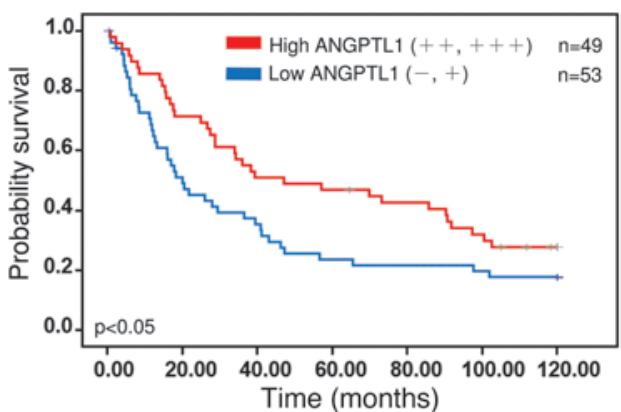

\section{Figure 1}

ANGPTL1 inversely correlates with stage, tumor status, lymph node status, invasion, and poor prognosis of lung cancer. (A) Staining with normal mouse IgG as negative control. Scale bar: $100 \mu \mathrm{m}$. (B) Strong staining of ANGPTL1 in normal lung bronchi. Scale bar: $100 \mu \mathrm{m}$. (C) Strong staining of ANGPTL1 (high expression). Scale bar: $100 \mu \mathrm{m}$. (D) Weak staining of ANGPTL1 (low expression). Scale bar: $100 \mu \mathrm{m}$. (E-H) Percentage of patients with high expression $(++,+++)$ of ANGPTL1 and low expression (-, + ) of ANGPTL1 according to different clinical parameters as follows: (E) tumor stage, (F) tumor status, (G) lymph node status, $(\mathbf{H})$ cancer type. Numbers in bars represent the percentage of patients for each condition. AC, invasive adenocarcinoma; BAC, bronchoalveolar carcinoma. ${ }^{*} P<0.05,{ }^{*} P<0.01$ ( $n=102$ [E-G]; $n=70$ [H]; 2-sided Pearson $\chi^{2}$ test). (I and $\mathbf{J}$ ) KaplanMeier plot of (I) overall and (J) disease-free survival of patients with lung cancer, stratified by ANGPTL1 expression. The logrank test was used to compare differences between groups $(n=102)$. addition of blocking antigen and is shown in Supplemental Figure 1A (supplemental material available online with this article; doi:10.1172/JCI64044DS1). ANGPTL1 expression was detectable in normal lung bronchi (Figure 1B); staining was not detected when normal mouse IgG was used (Figure 1A). Strong ANGPTL1 staining was observed in the cytoplasm in early-stage lung cancer (Figure 1C). In contrast, weak ANGPTL1 staining was observed in advanced-stage lung cancer (Figure 1D). ANGPTL1 expression inversely correlated with advanced-stage, higher grade tumor status and higher grade lymph node status (Figure 1, E-G). Notably, ANGPTL1 expression was higher in a subtype of adenocarcinoma, bronchoalveolar carcinoma, which showed a less invasive phenotype than adenocarcinoma (Figure $1 \mathrm{H}$ ). To study the significance of ANGPTL1 in lymph node metastasis, we collected 12 sets of primary lung tumors with matched lymph node metastatic tumors from the same patient. Interestingly, ANGPTL1 expression was significantly reduced in the lymph node metastatic tumors compared with that in primary lung tumors $(n=12)$ (Supplemental
Figure 1, B-D). We also found an inverse correlation between ANGPTL1 and lymph node metastasis in patients with breast cancer $(n=52)$ (Supplemental Figure 2, A-E). Additionally, we analyzed ANGPTL1 mRNA expression in data from a publically available GEO database $(19,20)$ and found that ANGPTL1 mRNA expression was higher in primary prostate tumors $(n=66)$ than in lymph node metastatic prostate tumors $(n=15)$ (Supplemental Figure 2F). Most importantly, patients with lung cancer with high ANGPTL1 expression had longer overall survival and disease-free survival (Figure 1, I and J). Taken together, these results indicate that ANGPTL1 inversely correlates with poor prognosis in patients with cancer.

ANGPTL1 inhibits cancer cell migration, invasion, and metastasis. To explore the effects of ANGPTL1 on the invasiveness of cancer cells, we examined the expression of ANGPTL1 in a panel of lung cancer cell lines, CL1-0 and CL1-5, which exhibited increasingly invasive behavior (ref. 21 and Figure 2A). We also established a similar panel of breast cancer cell lines, MDA- 
A
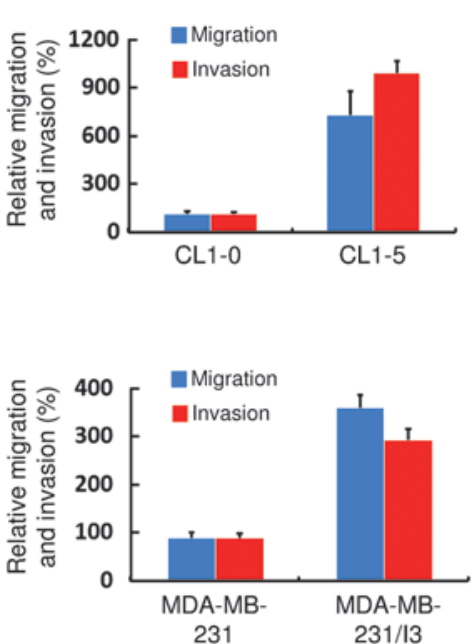

D
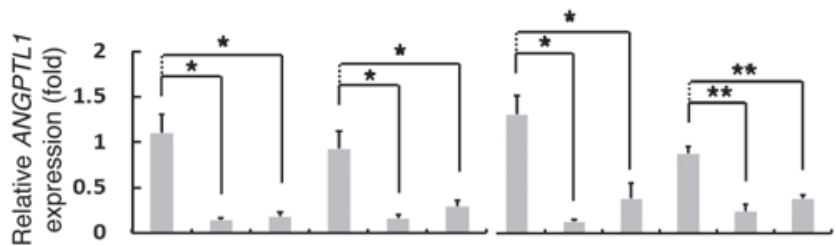

ShRNA-LUC

shRNA-ANGPTL1-1

shRNA-ANGPTL1-2
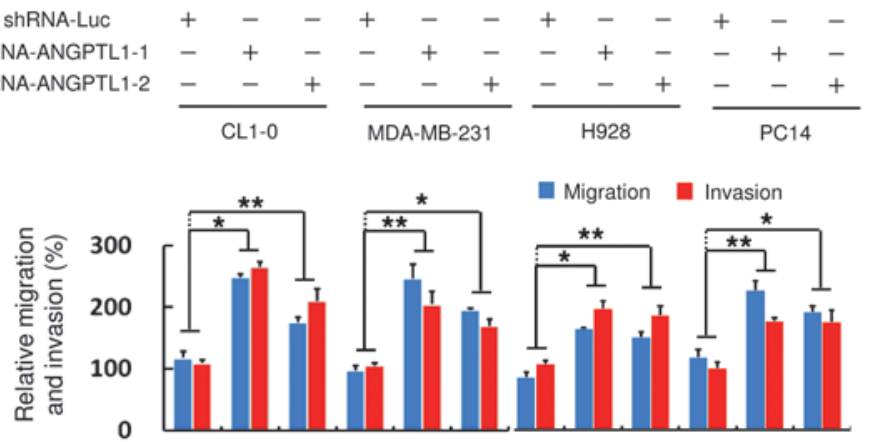

ShRNA-Luc ShRNA-ANGPTL1-1 ShRNA-ANGPTL1-2
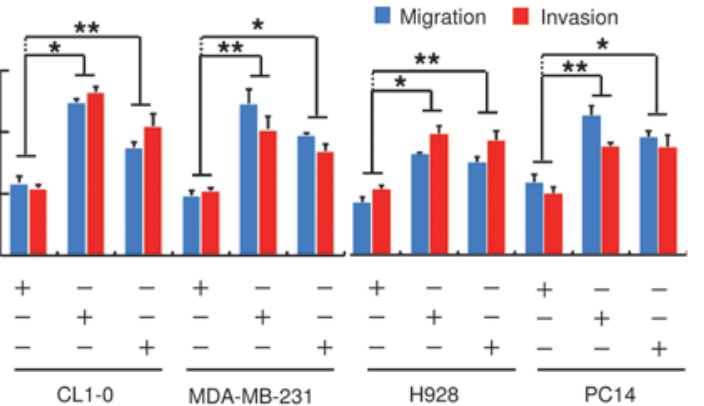

$\mathbf{F}$

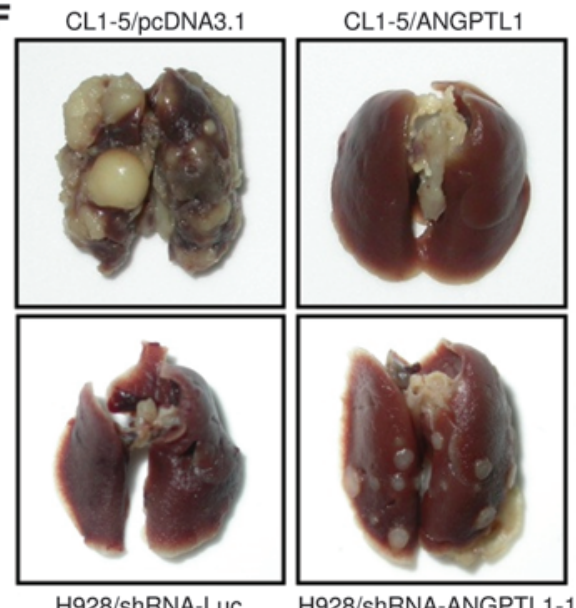

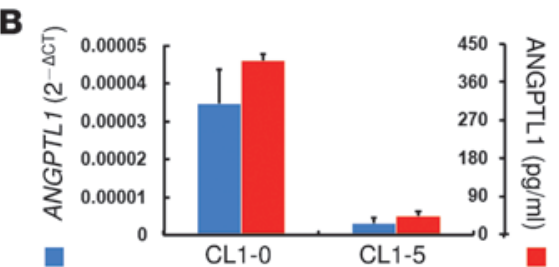

C
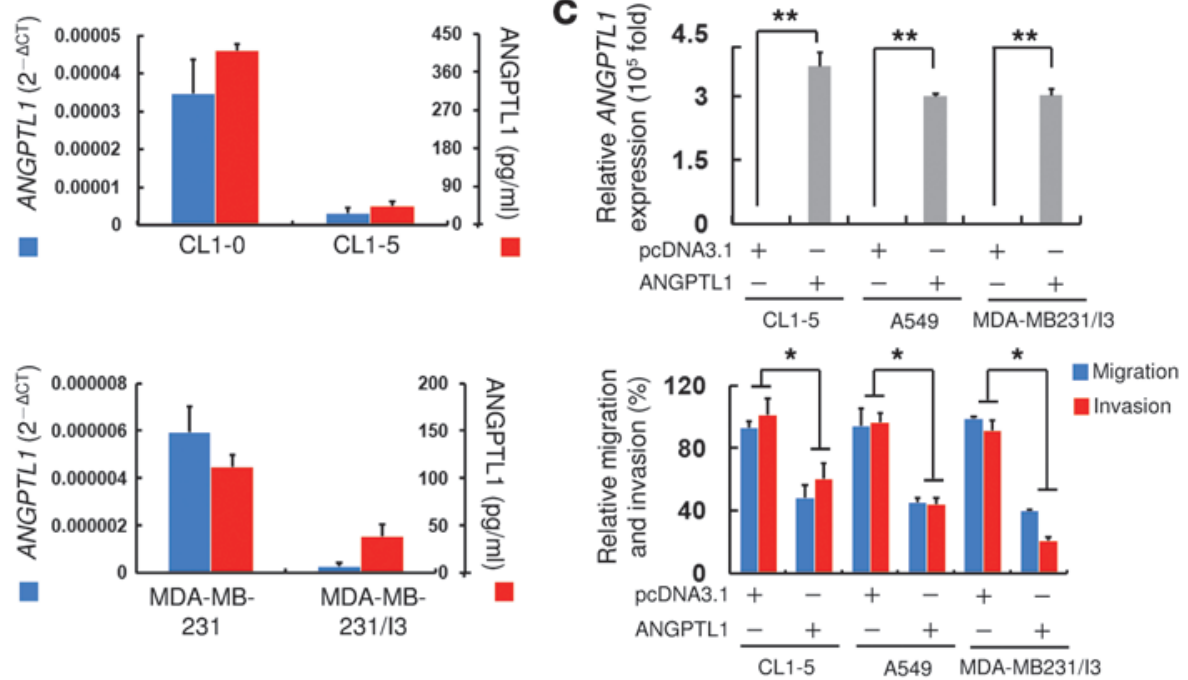

$\mathbf{E}$

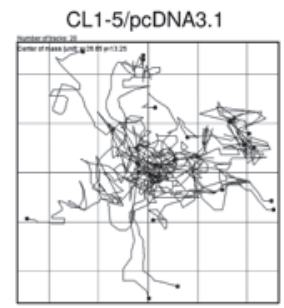

H928/shRNA-Luc
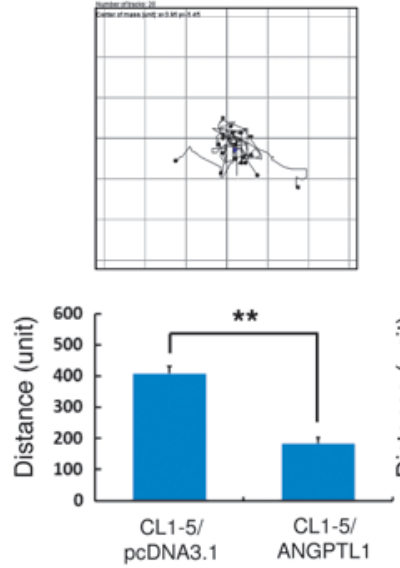

H

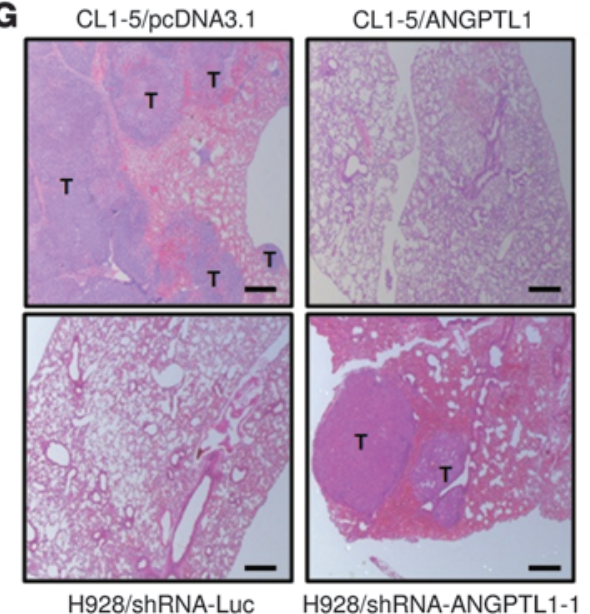

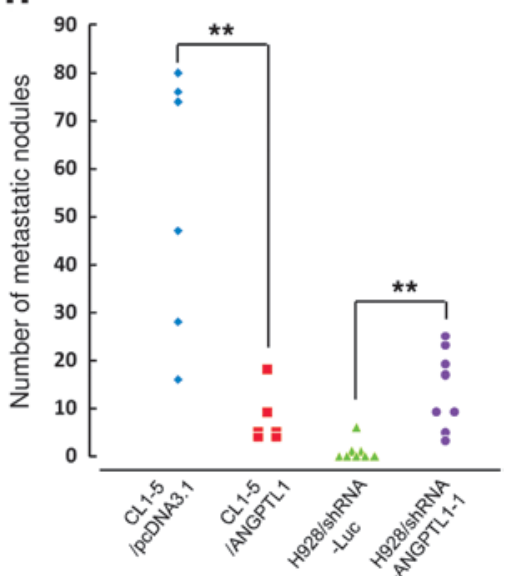




\section{Figure 2}

ANGPTL1 inhibits cancer cell motility and metastasis. (A) The relative migration and invasion and (B) the ANGPTL1 expression and secreted ANGPTL1 level in a series of cancer cells. (C and D) qRT-PCR analysis of ANGPTL1 and measurement of the migration and invasion in (C) ANGPTL1-overexpressing CL1-5, A549, and MDA-MB-231/I3 cells and (D) ANGPTL1 knockdown CL1-0, MDA-MB-231, H928, and PC14 cells. (E) Measurement of the relative migration in ANGPTL1-overexpressing CL1-5 and ANGPTL1 knockdown H928 cells. Representative images of time-lapse cell tracker migration assay (top). Quantification of timelapse cell tracker migration assay (bottom). $(\mathbf{F}-\mathbf{H})$ Measurement of the relative metastasis capability (tail vein injection) in ANGPTL1-overexpressing CL1-5 and ANGPTL1 knockdown H928 cells. (F) Representative images of lungs of mice. (G) Histological analyses of lung metastatic tumors by hematoxylin and eosin staining. $T$ indicates tumor sites. Scale bars: $500 \mu \mathrm{m}$. (H) ANGPTL1 overexpression and knockdown and total numbers of lung metastatic nodules. Data are shown as mean \pm SEM. ${ }^{*} P<0.05,{ }^{\star \star} P<0.01$ (2-tailed Student's $t$ test).

MB-231 and MDA-MB-231/I3 (Figure 2A). ANGPTL1 mRNA and secreted ANGPTL1 were highly expressed in parental cells (CL1-0 and MDA-MB-231) but were barely detectable in their derivative cells (CL1-5 and MDA-MB-231/I3) (Figure 2B). Similar results were also found in other cancer cell lines, including lung and breast cancers (Supplemental Figure 3, A and B). To further examine the effect of ANGPTL1 on the motility and invasiveness of cancer cells, we transfected ANGPTL1-expressing vectors or control vectors into highly invasive CL1-5, A549, and MDA-MB-231/I3 cells. Overexpression of ANGPTL1 significantly decreased the migratory and invasive capabilities of the lung and breast cancer cells, as assessed by transwell migration and Matrigel invasion assays (Figure 2C). In contrast, knockdown of ANGPTL1 in the less invasive CL1-0, MDA-MB-231, H928, and PC14 cells increased their migratory and invasive behaviors (Figure 2D). However, our results showed that cells overexpressing ANGPTL1 and cells with ANGPTL1 knockdown exhibited no significant differences in cell proliferation (Supplemental Figure 3C). Cell motility was also examined using the time-lapse cell tracker assay (Figure 2E) and wound healing assay (Supplemental Figure 3D). The results showed that ANGPTL1 significantly inhibits lung cancer cell migration. Treatment with conditional medium (CM) from HEK293T (293T) cells overexpressing ANGPTL1 (Supplemental Figure $3 \mathrm{E}$ ) and ANGPTL1 recombinant protein (rANGPTL1) (Supplemental Figure 3F) markedly inhibited the migratory and invasive abilities of lung cancer cells. Interestingly, treatment with a specific ANGPTL1 antibody to block the effect of 293T/ ANGPTL1 CM could recover the inhibitory migration and invasion (Supplemental Figure 3E). These data suggest that the ANGPTL1 plays an important role in the inhibition of lung and breast cancer cell motility and invasiveness. To further investigate the role of ANGPTL1 in an in vivo model, we injected stable transfected cell lines (CL1-5/pcDNA3.1, CL1-5/ANGPTL1, H928/shRNA-Luc, and H928/shRNA-ANGPTL1-1) into the lateral tail vein of SCID mice and detected the lung metastatic nodules. Mice injected with CL1-5/pcDNA3.1 and H928/shRNA-ANGPTL1-1 cells had more and larger lung metastases than those injected with CL1-5/ANGPTL1 and H928/shRNA-Luc cells, respectively (Figure 2, F-H, and Supplemental Figure 3G). Histologic analyses (Figure 2G) and RT-PCR (Supplemental Figure $3 \mathrm{H}$ ) of lung dissected from each mouse confirmed lung metastases and ANGPTL1 expression, respectively. These results demonstrate that ANGPTL1 is crucial for suppression of lung and breast cancer cell invasion and metastasis.

ANGPTL1 induces MET by downregulating SLUG. We next wanted to identify how ANGPTL1 regulates the migratory and invasive phenotypes of cancer cells. In phase-contrast images, we found that the CL1-5/ANGPTL1 cells appeared round, with a more epithelial morphology than that of CL1-5/pcDNA3.1 cells (Figure 3A). In contrast, CL1-0/shRNA-ANGPTL1-1 cells showed a spindle-like, mesenchymal morphology, one of the major characteristics of EMT (Figure 3A). Similar results were also found in breast cancer cell lines (Supplemental Figure 4A). EMT is a critical step in metastasis that involves diverse biological mechanisms (11). We further investigated the expression of EMT markers and EMT-related transcription factors using Western blot analysis. Our data showed that ANGPTL1 increases the expression of the epithelial marker E-cadherin and represses the expression of mesenchymal markers ( $\mathrm{N}$-cadherin, fibronectin, and vimentin) and SLUG protein in lung and breast cancer cell lines (Figure 3, B and C; Supplemental Figure 4, B and C; and Supplemental Figure 6A). Interestingly, ANGPTL1 affects SLUG protein expression but not SLUG mRNA expression (Supplemental Figure 6, B-D). Moreover, we found that EMT markers and the migratory and invasive capabilities of cancer cells were restored by ectopic expression of SLUG in CL1-5/ANGPTL1 cells (Figure 3, D and E). In contrast, the shANGPTL1-induced migratory and invasive capabilities were repressed by knockdown of SLUG (Supplemental Figure 5, A and B). We next investigated the correlation between the expression of ANGPTL1 and SLUG in a cohort of 102 patients with lung cancer. Immunohistochemical staining results revealed that ANGPTL1 expression inversely correlated with SLUG expression (Supplemental Figure 6, E and F). To determine whether these results were reproducible in metastasis in vivo, we established GFP- and luciferase-expressing CL1-5 cells (CL1-5GL cells) and transfected them with pcDNA3.1 and ANGPTL1 in combination with pLKO_AS2.neo and PLKO_AS2. neo/SLUG (Figure 3F). The cells were orthotopically injected into the upper lobes of the left lungs and lateral tail veins of SCID mice. Tumor metastasis was monitored by bioluminescence imaging. In the lung orthotopic model, tumor metastasis from the left to right lung was decreased by ANGPTL1 and significantly recovered by SLUG overexpression (Figure 3, G-I, top, and Supplemental Figure 4D). Overexpression of SLUG also recovered lung metastatic activity in CL1-5GL/ANGPTL1 cells (Figure 3, G-I, bottom, and Supplemental Figure 4E) in the tail vein model. The metastatic capabilities of CL1-0/shRNA-ANGPTL1-1 cells were reduced by knockdown of SLUG (Supplemental Figure $5, \mathrm{C}-\mathrm{G})$. Taken together, these results indicate that SLUG is an important downstream effector of ANGPTL1-mediated suppression of lung cancer cell invasiveness and metastasis.

ANGPTL1 represses SLUG expression by inducing expression of miR630. SLUG degradation may lead to the aberrant SLUG accumulation found in several tumors (22-24). Because the levels of SLUG protein were dramatically changed by altered ANGPTL1 expression, we examined the effects of ANGPTL1 on SLUG protein stability. CL1-5/pcDNA3.1 and CL1-5/ANGPTL1 cells were treated with MG132 (26S proteasome inhibitor), and the SLUG protein expression levels were determined. ANGPTL1-mediated reduction of SLUG protein expression was not restored by treatment with MG132 (Figure 4A). Furthermore, in the cyclohexi- 
A $\mathrm{CL} 1-5 / \mathrm{pcDNA3.1}$

CL1-5/ANGPTL1
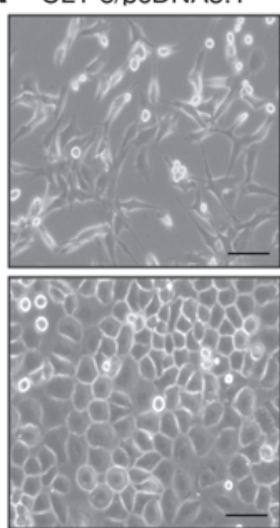

CL1-0/shRNA -Luc

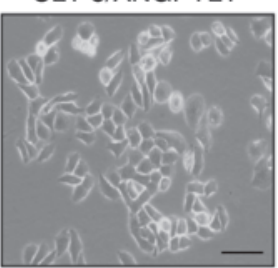

CL1-0/shRNA -ANGPTL1-1
$\mathbf{B}$

E-cadherin

$\mathrm{N}$-cadherin

Fibronectin

Vimentin

$\alpha$-Tubulin

\section{E}
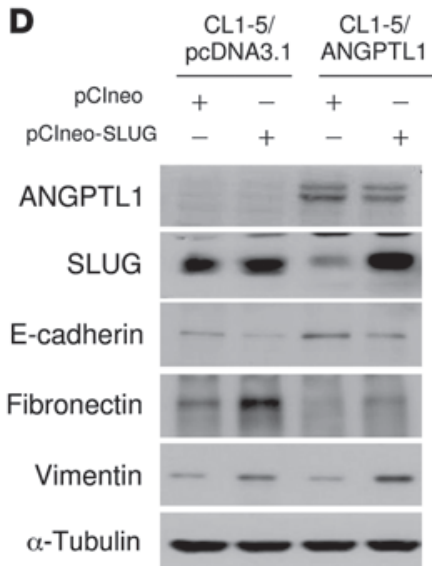

G Lung orthotopic injection

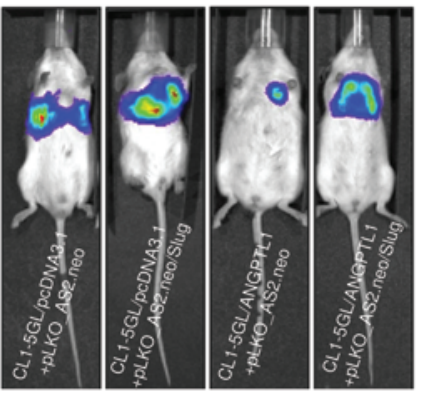

$\begin{array}{llll}2.0 & 1.5 & 1.0 & 0.5\end{array}\left(\times 10^{\prime}\right)$

$\mathrm{p} / \mathrm{sec} / \mathrm{cm}^{2} / \mathrm{si}$

Tail vein injection

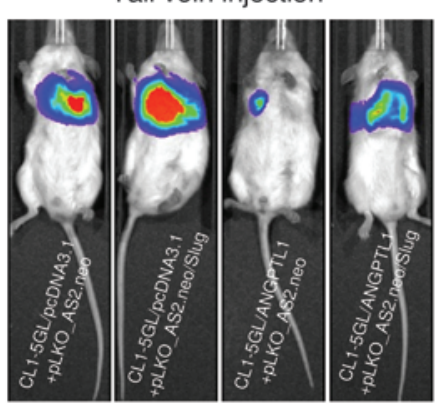

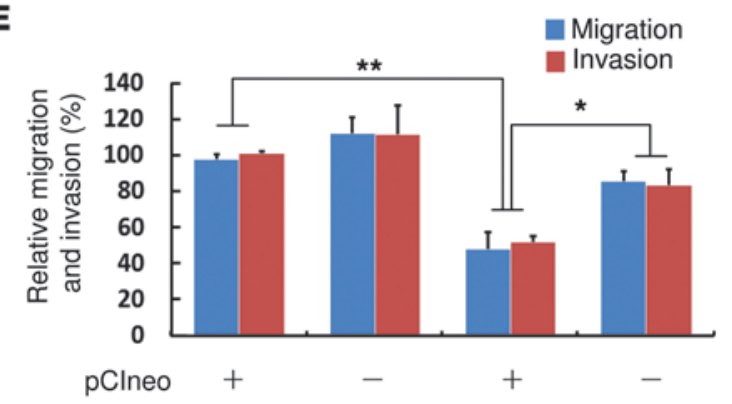

pCIneo-SLUG

$$
\text { CL1-5/pcDNA3.1 }
$$

CL1-5/ANGPTL1

H

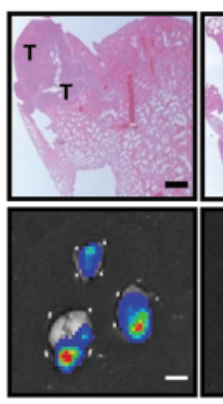

Lung orthotopic injection

CL1-5GL/pcDNA3.1 CL1-5GL/pcDNA3.1 CL1-5GL/ANGPTL1 CL1-5GL/ANGPTL1 $\mathrm{cm}^{2} / \mathrm{si}$ +pLKO_AS2.neo +pLKO_AS2.neo/SLUG +pLKO_AS2.neo +pLKO_AS2.neo/SLUG

Tail vein injection

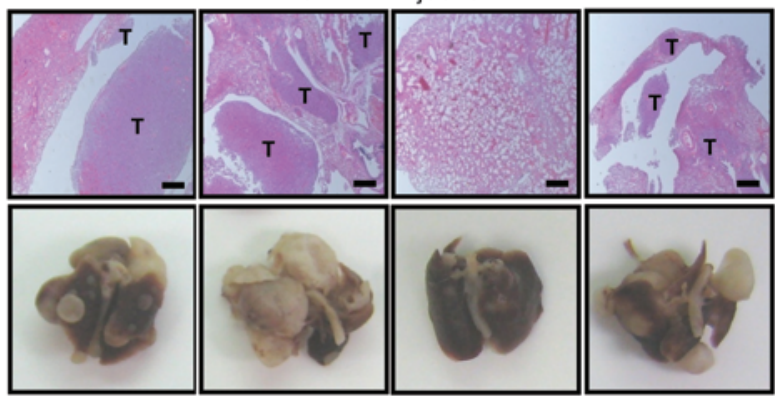

CL1-5GL/pcDNA3.1 CL1-5GL/pcDNA3.1 CL1-5GLANGPTL1 CL1-5GLANGPTL1 +pLKO AS2.neo +pLKO_AS2.neo/SLUG +pLKO_AS2.neo +pLKO AS2.neo/SLUG

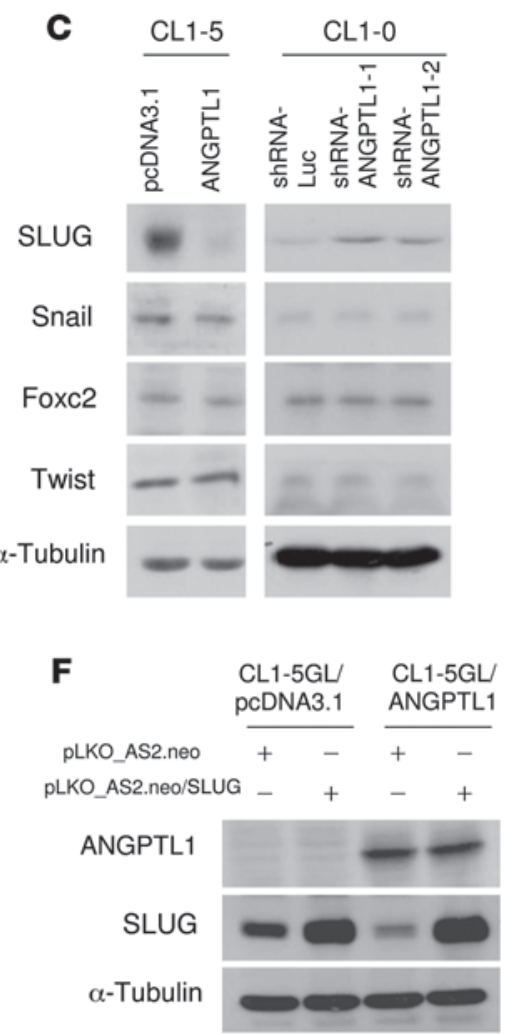

I Lung orthotopic injection

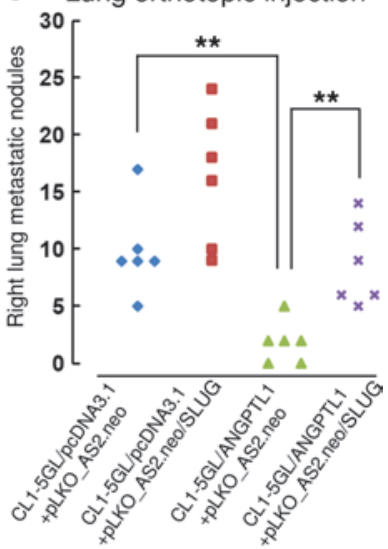

Tail vein injection

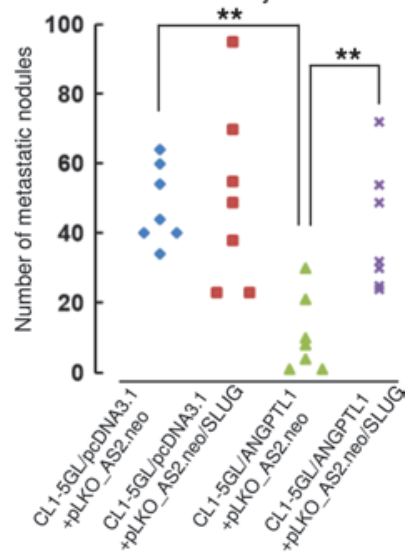




\section{Figure 3}

ANGPTL1 represses EMT by targeting SLUG in lung cancer. (A) Phase-contrast images of ANGPTL1-overexpressing CL1-5 cells and ANGPTL1 knockdown CL1-0 cells. Scale bars: $100 \mu \mathrm{m}$. (B) Western blot analysis of $\mathrm{E}$-cadherin, $\mathrm{N}$-cadherin, fibronectin, and vimentin in ANGPTL1-overexpressing CL1-5 cells and ANGPTL1 knockdown CL1-0 cells. (C) Western blot analysis of SLUG, Snail, Foxc2, and Twist in ANGPTL1-overexpressing CL1-5 cells and ANGPTL1 knockdown CL1-0 cells. (D) Western blot analysis of ANGPTL1, SLUG, E-cadherin, fibronectin, and vimentin and (E) measurement of the migration and invasion in ANGPTL1 in ANGPTL1-overexpressing CL1-5 cells transiently transfected with pCIneo and pCIneo-SLUG. (F) Western blot analysis of ANGPTL1 and SLUG in ANGPTL1-overexpressing CL1-5 cells infected with pLKO_AS2.neo and pLKO_AS2.neo/SLUG. (G) Representative luciferase images of mice: lung orthotopic injection (top); tail vein injection (bottom). (H) Lungs were isolated and examined after lung orthotopic injection (top panel) and after tail vein injection (bottom panel). Histological analyses of lung metastatic tumors by hematoxylin and eosin staining (top rows). Representative luciferase activity images and images of lungs of mice (bottom rows). Scale bars: $500 \mu \mathrm{m}$ (top row, top and bottom panel); 5 um (bottom row, top panel). (I) The numbers of lung metastatic nodules: lung orthotopic injection (top); tail vein injection (bottom). Data are shown as mean \pm SEM. ${ }^{\star} P<0.05,{ }^{* \star} P<0.01$ (2-tailed Student's $t$ test).

mide pulse-chase experiment, the half-life of the SLUG protein was not significantly different between CL1-5/pcDNA3.1 and CL1-5/ANGPTL1 cells (Figure 4B). Overexpression of ANGPTL1 in a breast cancer cell line, MDA-MB-231/I3, resulted in consistent findings (Supplemental Figure 7, A and B). These results suggest that SLUG protein stability is not affected by ANGPTL1, indicating that ANGPTL1-modulated MET processes likely occur through posttranscriptional regulation of SLUG. Several studies have shown that miRNAs are involved in the regulation of EMT and cancer progression (25-28). To investigate whether ANGPTL1 could regulate miRNA expression, we analyzed global miRNA expression in CL1-5/pcDNA3.1 and CL1-5/ANGPTL1 cells using miRNA microarray analysis. We integrated the miRNA microarray result(s) with 3 online computational algorithms (TargetScan, PicTar, and miRanda) and filtered out 10 candidate miRNAs (Figure 4C). We confirmed these candidates using quantitative RT-PCR (qRT-PCR) in CL1-5/ANGPTL1 and CL1-0/shANGPTL1-1 cells and their control cells (Figure 4D). Two candidate miRNAs, miR-545 and miR-630, were upregulated by ANGPTL1 in CL1-5 cells and downregulated by shANGPTL1 in CL1-0 cells. To examine whether miR-545 and miR-630 regulate the 3'-UTR of SNAI2 (the SLUG transcripts), we constructed luciferase reporter vectors harboring the wild-type 3 '-UTR of the SNAI2 mRNA (WT-3'-UTR) and vectors containing mismatches in the predicted miR-545- and miR-630-binding site (MT-3'-UTR; Figure 4E) and transfected these vectors into $293 \mathrm{~T}$ cells at different miRNA-to-reporter ratios. Transfection of the WT-3'-UTR plasmid, but not the MT-3'-UTR plasmid, resulted in suppression of luciferase activity in a dose-dependent manner. This result indicates that miR-545 and miR-630 have a negative effect on SNAI2 3'-UTR expression; miR-630 had more significant repressive effects (Figure 4E). We also detected the expression of miR-545 and miR-630 in CL1-5 cells treated with rANGPTL1. The results showed that the expression of miR-545 and miR-630 was significantly induced after rANGPTL1 treatment (Figure 4F). Blockage of miR-545 and miR-630 expression using miRNA inhibitors resulted in a significant reduction of miR-545 and miR-630 expression in CL1-5/ANGPTL1 cells (Supplemental Figure 7, C and D). To further test whether miR-545 and miR-630 repressed SLUG protein expression, we introduced miR-545 and miR-630 inhibitors into CL1-5/ANGPTL1 cells and performed Western blot analysis. Interestingly, anti-miR-630 obviously restored SLUG protein expression (Figure 4H, top) but not SLUG mRNA expression (Supplemental Figure 7E). Anti-miR-545 had slight effects on SLUG protein expression (Figure 4G), and this result was consistent with the reporter assay in which miR-545 had fewer suppressive effects on SNAI2 3'-UTR expression. Moreover, we found that migration and invasion of cancer cells were significantly restored by introduction of anti-miR-630, but not anti-miR-545, into CL1-5/ANGPTL1 cells (Figure 4, G and H). We then investigated whether ANGPTL1 or SLUG expression correlated with miR-630 expression in a cohort of 73 patients with lung cancer. qRT-PCR results revealed that ANGPTL1 protein expression positively correlated with miR-630 expression; SLUG protein expression inversely correlated with miR-630 expression (Supplemental Figure 8, A and B). We evaluated the clinical implications of miR-630 in this cohort. Interestingly, miR-630 expression inversely correlated with advanced-stage, higher grade lymph node status, invasion, poor overall survival, and poor diseasefree survival (Supplemental Figure 8, C-G). To understand the mechanism of ANGPTL1-induced miR-630 expression, we analyzed pre-miR-630 and its precursor pri-miR-630 expression in CL1-5/ANGPTL1 and CL1-0/shANGPTL1-1 cells and their control cells. The data showed that ANGPTL1 significantly increases pre-miR-630 and pri-miR-630 expression (Supplemental Figure 9, A and B). This result suggested that ANGPTL1 regulates miR-630 expression at the transcriptional level. Together, these results indicate that miR-630 is an important modulator of both ANGPTL1-mediated SLUG suppression and inhibition of lung cancer cell motility and invasiveness.

ANGPTL1 interacts with integrin $\alpha_{1} \beta_{1}$ and represses the downstream signaling and SLUG expression. Several studies have shown that ANGPTL proteins deliver their signals via integrin receptorrelated pathways (29-33). We assumed, therefore, that ANGPTL1mediated SLUG expression might occur through integrin signaling pathways. We used an ECM cell adhesion array to analyze what kind of ECM is essential for the adhesion of CL1-5/pcDNA3.1 and CL1-5/ANGPTL1 cells and to identify candidate integrin receptors for ANGPTL1. Interestingly, overexpression of ANGPTL1 significantly reduced cell adhesion to collagen-, fibronectin-, and vitronectin-coated wells (Supplemental Figure 10). We examined whether ANGPTL1 interacted with specific integrins using immunoprecipitation. Analysis of immunoprecipitates from CL1-5 cells treated with V5-tagged ANGPTL1 CM revealed that ANGPTL1 interacts with the integrin $\alpha_{1}$ and $\beta_{1}$ (Figure 5A). ELISA and surface plasmon resonance results also confirmed the interaction of ANGPTL1 with integrin $\alpha_{1} \beta_{1}$ (Figure 5, B and C). Previous studies showed that the fibrinogen-like domain (FD) of ANGPTLs is sufficient for integrin binding $(31,32)$. We defined the coiledcoil domain (CCD) and FD of ANGPTL1 by Human Protein Reference Database (http://www.hprd.org/) (Supplemental Figure 11A). We found that the FD of ANGPTL1 is essential for binding to integrin $\alpha_{1}$ and $\beta_{1}$ (Supplemental Figure 11B). To further investigate the integrin signaling associated with the ANGPTL1-inhibited SLUG, we examined the effects of ANGPTL1 on the activity of integrin $\beta_{1}$ using a specific antibody (34) and downstream signal 
A

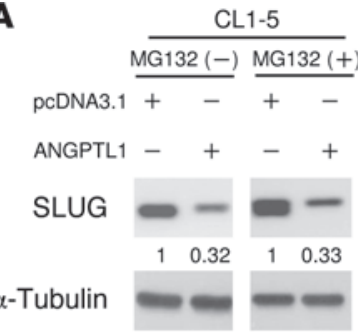

B

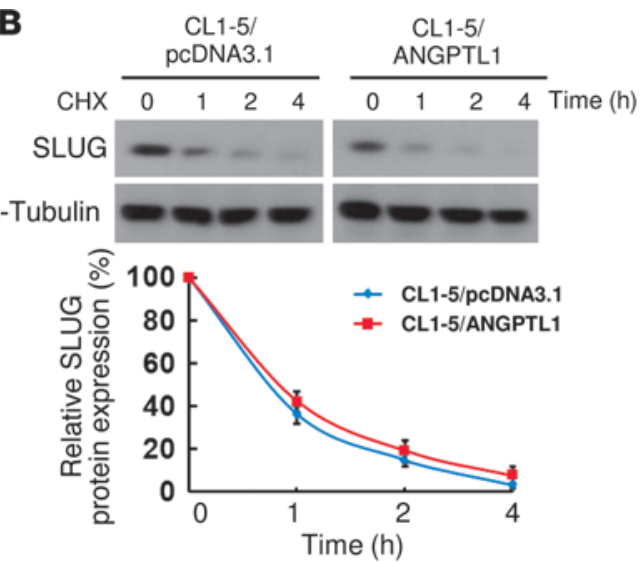

E

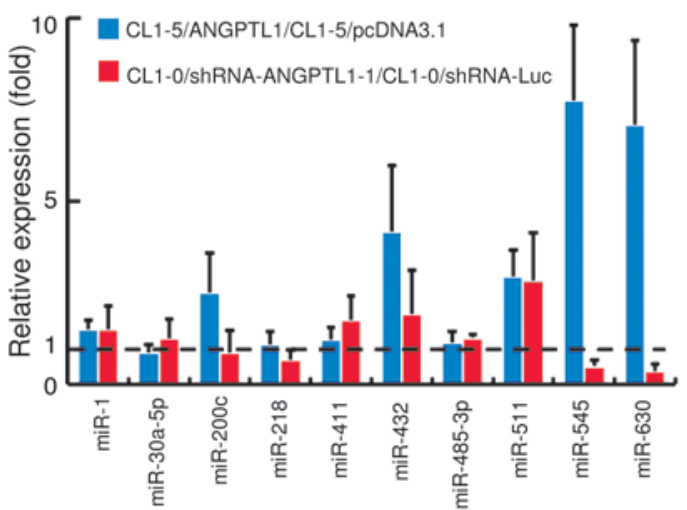

3'GUUAUUUACAAACGACU 5' hsa-miR-545 |IIIII 5'UACUAUAUAUUUGCUGA $3^{\prime}$ H.S. 3UTR of SNAI2 wild type (WT) sequence 5'UACUAUAUAGGGGGGGA $3^{\prime}$ H.S. 3UTR of SNAI2 mutant type (MT) 5' UGUUUACUCAAAAAAAA 3' H.S. 3UTR of SNAI2 mutant type (MT: $5^{\prime}$ UACUAUAUAGGGGGGGA
$\frac{\begin{array}{c}\text { Seed } \\ \text { sequence }\end{array}}{}$
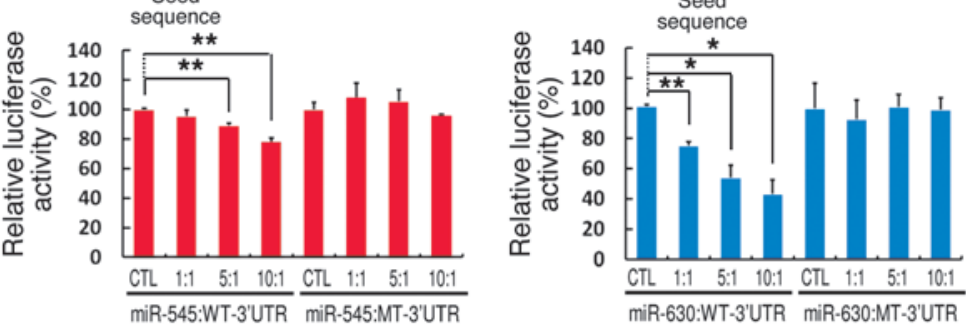

$$
\text { 5' UACUCGAACAGAAUGCA } 3^{\prime} \text { H.S. 3UTR of SNAI2 wild type (WT) }
$$
miR-630:WT-3'UTR miR-630:MT-3'UTR

$\mathbf{F}$

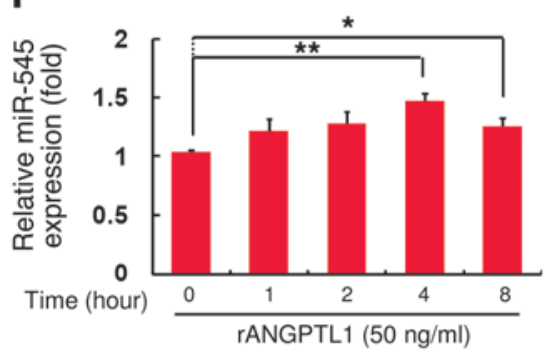

G
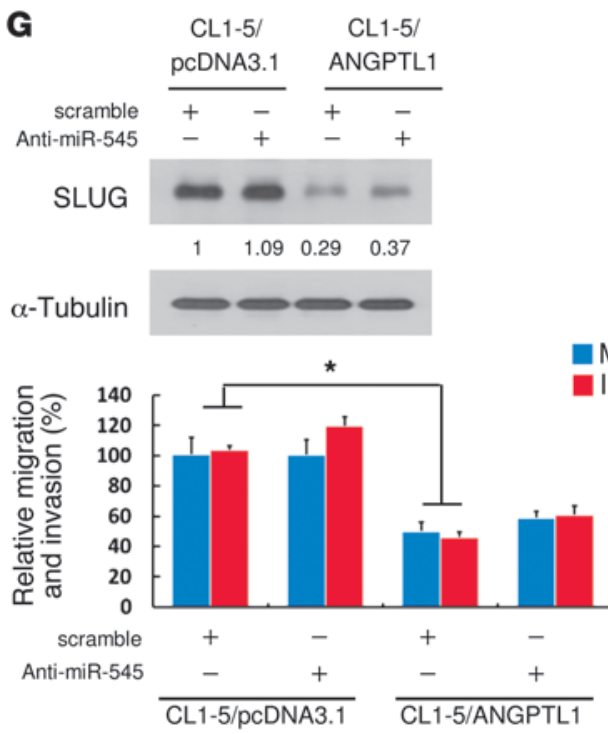

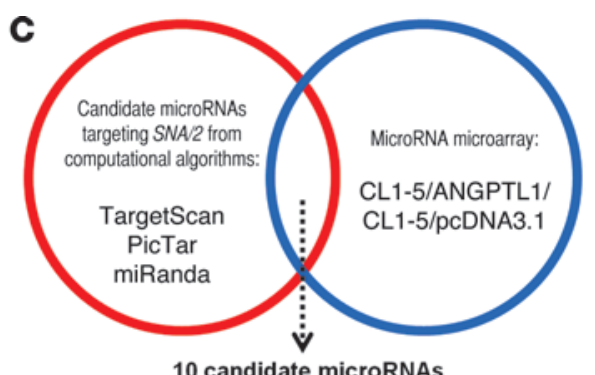

3'GGGACCAUGUCUUAUGA 5' hsa-miR-630
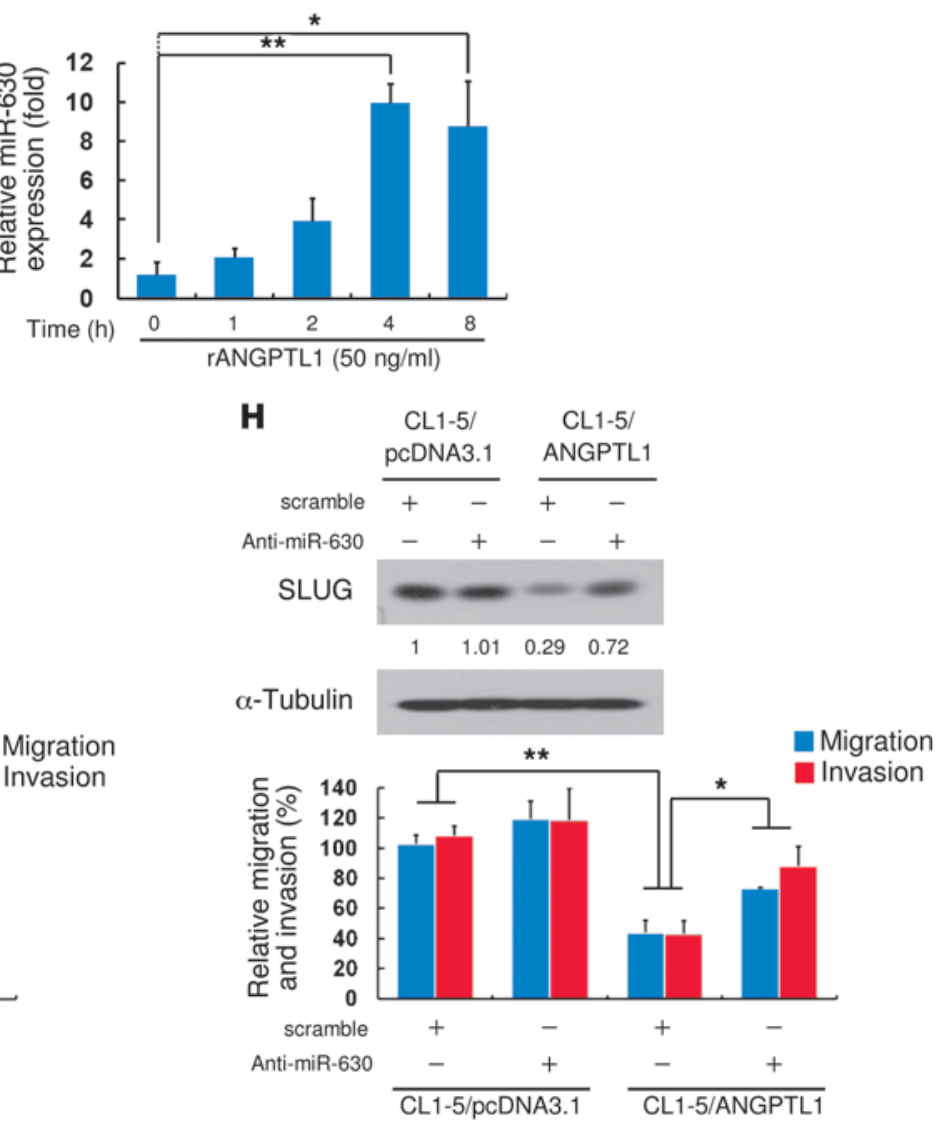


\section{Figure 4}

ANGPTL1 represses SLUG protein expression through inducing miRNA-630. (A) Western blot analysis of SLUG in ANGPTL1-overexpressing CL1-5 cells after treatment with $10 \mu \mathrm{M}$ MG132. SLUG levels were arbitrarily assigned a value of 1 in CL1-5/pcDNA3.1 cells. Numbers above the blots represent SLUG levels. (B) Western blot analysis of SLUG in ANGPTL1-overexpressing CL1-5 cells after treatment with $10 \mu \mathrm{g} / \mathrm{ml}$ cycloheximide (CHX). SLUG levels of CL1-5/pcDNA3.1 and CL1-5/ANGPTL1 were arbitrarily assigned a value of $100 \%$ at 0 hours. (C) Schematic selection of candidate miRNAs. Using overexpression of ANGPTL1 in CL $1-5$ cells as a model in miRNA microarrays, 10 candidate miRNAs (merged microarray data with software predictive results from 3 online computational algorithms, TargetScan, PicTar, and miRanda) were selected. (D) qRT-PCR analysis of differential expression of 10 miRNAs in ANGPTL1-overexpressing CL1-5 cells and ANGPTL1 knockdown CL1-0 cells. The dashed line indicates that the qPCR value of CL1-5/ANGPTL1 was divided by CL1-5/pcDNA3.1. (E) Schematic diagram presents the predicted miR-545- and miR$630-$ binding sequences or mutated versions (top). Luciferase activity (bottom) of wild-type SNAI2 3'-UTR (WT-3'-UTR) or mutated-type SNAI2 3'-UTR (MT-3'-UTR) reporter genes in 293T cells transfected with miR-545 and miR-630 at different ratios. (F) qRT-PCR analysis of miR-545 and miR-630 expression in CL $1-5$ cells treated with $50 \mathrm{ng} / \mathrm{ml}$ rANGPTL1. ( $\mathbf{G}$ and $\mathbf{H}$ ) Western blot analysis of SLUG and measurement of the migration and invasion in ANGPTL1-overexpressing CL1-5 cells transiently transfected with $(\mathbf{G})$ anti-miR-545 and $(\mathbf{H})$ anti-miR630. Numbers above the blots represent SLUG levels. Data are shown as mean \pm SEM. ${ }^{*} P<0.05,{ }^{* *} P<0.01$ (2-tailed Student's $t$ test).

transduction pathways. The expression of activated integrin $\beta_{1}$ was repressed by ANGPTL1 overexpression (Figure 5D). For the downstream signaling, our results showed that phosphorylated FAK, ERK, and AKT were repressed by ANGPTL1 (Figure 5, E and F). To confirm the role of ERK and AKT signaling pathways in the regulation of SLUG, we transfected vectors expressing constitutively activated MEK1 (to activate ERK signal) and constitutively activated AKT (myr-AKT) into CL1-5/ANGPTL1 cells. We found that cancer cell migration and invasion and SLUG protein levels were restored by MEK1 (Figure 6B) but not by myr-AKT (Figure 6A). Next, we investigated the correlation between the expression of ANGPTL1 and p-ERK in patients with lung cancer $(n=27)$. Western blot results showed that expression of ANGPTL1 inversely correlated with p-ERK expression (Supplemental Figure 12, A and B). Two important collagen receptors, integrin $\alpha_{1} \beta_{1}$ and integrin $\alpha_{2} \beta_{1}$, have been shown to correlate with cell invasiveness and metastasis (35-39). To evaluate whether integrin $\alpha_{1} \beta_{1}$ is involved in ANGPTL1-regulated cell motility and invasiveness and SLUG expression, we first investigated the expression of integrin $\alpha_{1} \beta_{1}$ and integrin $\alpha_{2} \beta_{1}$ using RT-PCR. We found that integrin $\alpha_{1}$, integrin $\alpha_{2}$, and integrin $\beta_{1}$ expression levels were not altered after overexpression of ANGPTL1 in CL1-5 cells (Supplemental Figure 13, A and B). Moreover, we introduced specific shRNAs for each integrin (integrin $\alpha_{1}$, integrin $\alpha_{2}$, and integrin $\beta_{1}$ ) and found that knockdown of integrin $\alpha_{1}$ and $\beta_{1}$ in CL1-5 cells abolished rANGPTL1-mediated reduction of cancer cell motility and invasiveness and SLUG expression (Figure 6, C and D). To further confirm the role of an outside-in signaling pathway in the regulation of ERK and miR-630, we treated CL1-5/integrin $\alpha_{1} \beta_{1}$ knockdown cells with rANGPTL1 and found that the levels of phosphorylated ERK and miR-630 were not altered (Figure 6, E and F). Therefore, we concluded that ERK and miR-630 are involved in the regulation of SLUG through an ANGPTL1/integrin $\alpha_{1} \beta_{1}$ signaling axis.
ANGPTL1 induces miR-630 transcription through repression of the ERK/ SP1 pathway. To define the relationship between the ERK signaling pathway and miR-630 expression, we introduced MEK1- and myrAKT-expressing vectors into CL1-5/ANGPTL1 cells. The results revealed that MEK1, but not myr-AKT, represses the expression of miR-630 (Figure 7A). Moreover, ANGPTL1-induced expression of pri-miR-630 and pre-miR-630 was also inhibited by MEK1 (Figure 7B). These results show that ERK signaling is essential for miR-630 transcription. To investigate the transcriptional regulation of miR630 by ANGPTL1, promoter reporter assays were performed. Interestingly, miR-630 is located in exon 14 of the ARIH1 gene (ariadne homolog, ubiquitin-conjugating enzyme E2 binding protein, 1); representative regions approximately $1.2-\mathrm{kb}$ upstream of the transcriptional initiation site of the miR-630 were investigated. To further determine which response element participates in the regulation of miR-630 promoter activity in response to ANGPTL1, we developed a series of miR-630 promoter reporter constructs (Figure $7 \mathrm{C}$, left). We found that the region of the miR-630 promoter from -137 to -92 bp was critical for ANGPTL1-mediated transcriptional regulation of miR-630 (Figure 7C, right). A database search for known sequences within the miR-630 promoter ( -137 to $-92 \mathrm{bp}$ ) using AliBaba 2.1 indicated the presence of binding sites for SP1, C/EBP $\alpha$, ENKTF-1, and HBP-1b (Figure 7D, top). SP1 and C/EBP $\alpha$ are known to be phosphorylated by various kinases, including MEK1/ERK $(40,41)$. We next investigated whether SP1 or C/EBP $\alpha$ associates with the miR-630 promoter through the MEK1/ERK pathway using ChIP assay. We found that SP1, not C/EBP $\alpha$, interacts with the miR-630 promoter through the MEK1/ERK pathway (Figure 7D, bottom). We also confirmed that this response element participates in ANGPTL1-regulated miR-630 promoter activity by the SP1 mutant construct (Figure 7E). These results showed that SP1-binding element in the miR-630 promoter region (-137 to -92 $\mathrm{bp}$ ) is essential for ANGPTL1-induced miR-630 promoter activity (Figure 7E). Furthermore, we established CL1-5/pcDNA3.1 and CL1-5/ANGPTL1 cell lines with SP1 and C/EBP $\alpha$ knockdowns. Knockdown of SP1, but not C/EBP $\alpha$, induced miR-630 expression and repressed SLUG expression (Figure 7F and Supplemental Figure 14). These results suggest that ANGPTL1-induced miR-630 transcription is dependent on repression of the ERK/SP1 pathway.

\section{Discussion}

ANGPLT1 is reportedly an antiangiogenic protein, suppressing VEGF/bFGF-induced proliferation, migration, tube formation, and adhesion of endothelial cells in vitro (42). The influence of ANGPLT1 on tumor growth suppression has been shown in fibrosarcoma and mouse melanoma cell lines, in which tumors from cells overexpressing ANGPTL1 are smaller than those from control cells $(9,42)$. These results suggest that ANGPTL1 represses tumor-associated angiogenesis. Here, using genetic modulation of ANGPTL1 and rANGPTL1 treatment in cancer cells, we found that ANGPTL1 effectively suppresses the migratory, invasive, and metastatic capabilities of lung and breast cancer cells. Moreover, our results demonstrate the clinical significance of ANGPTL1, as its expression inversely correlates with malignant phenotypes and directly correlates with longer patient survival. Thus, multiple lines of evidence indicate that ANGPTL1 has a suppressive effect on lung and breast tumor cells, suggesting that downregulation of ANGPTL1 is a crucial step in lung and breast cancer progression.

SLUG is overexpressed in numerous cancers and also promotes invasion in lung adenocarcinoma, glioma, ovarian, and pancreatic 
A

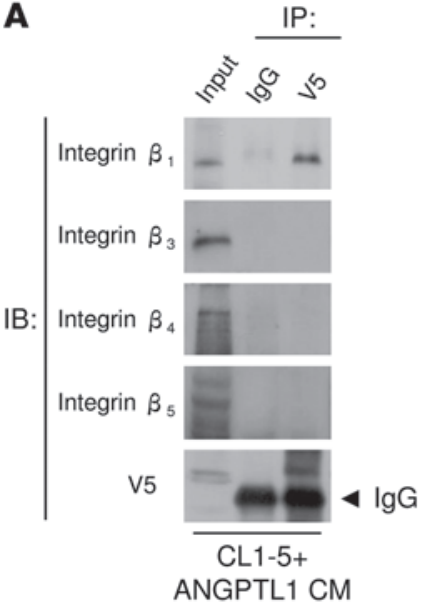

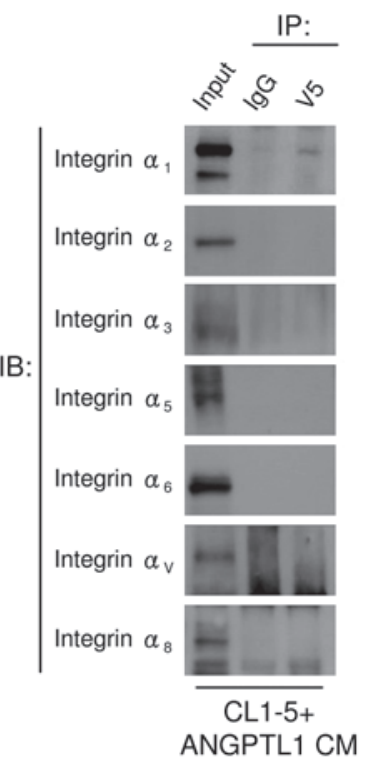
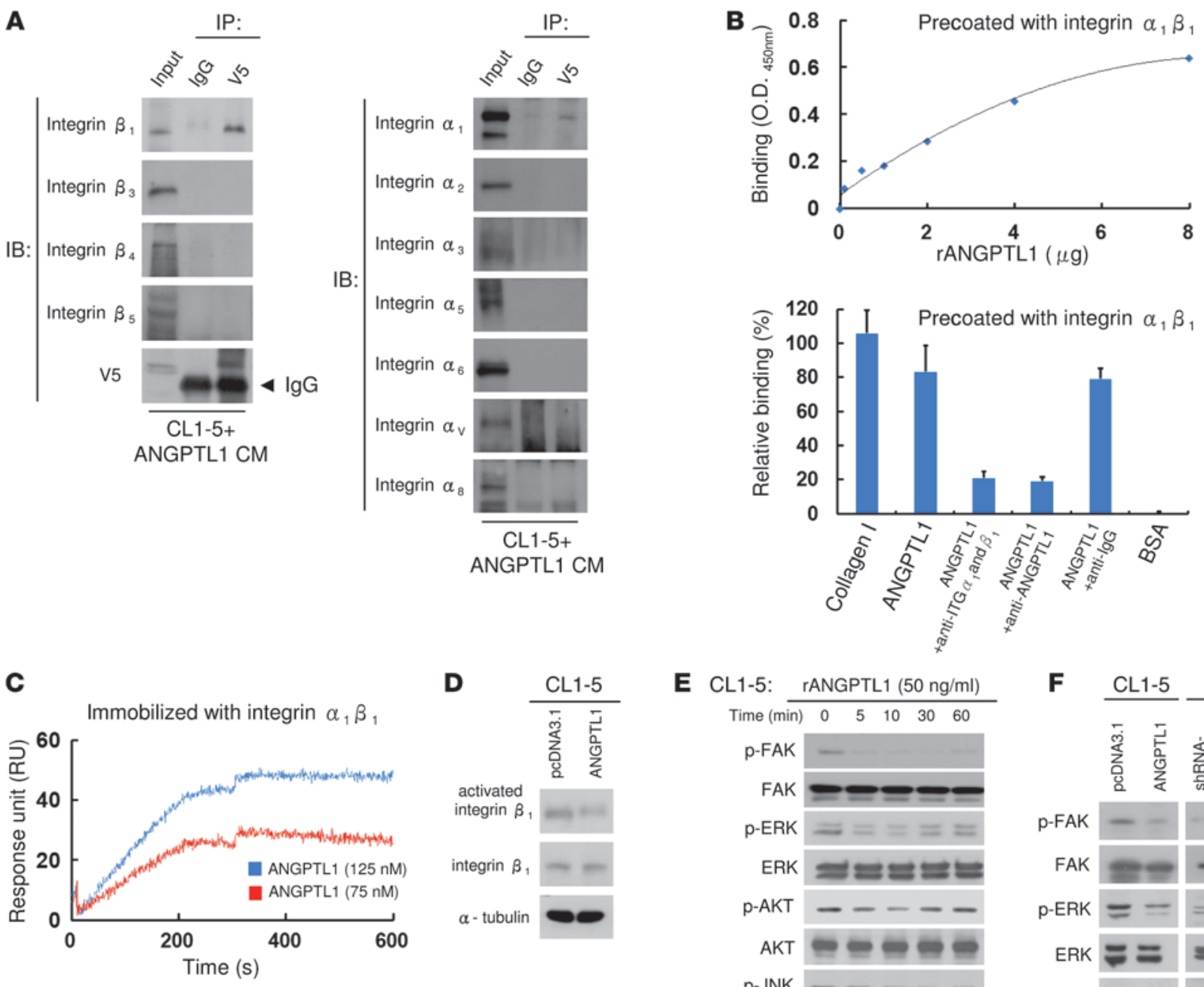

E

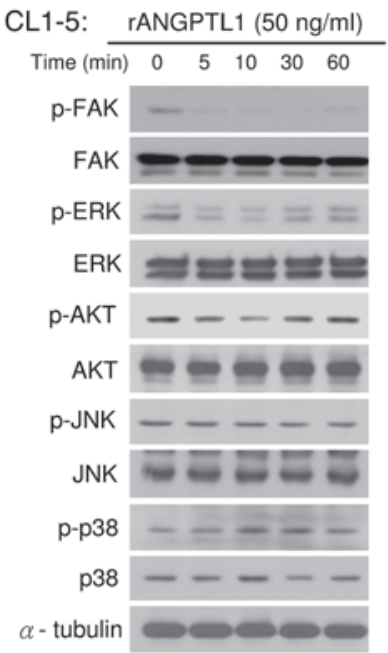

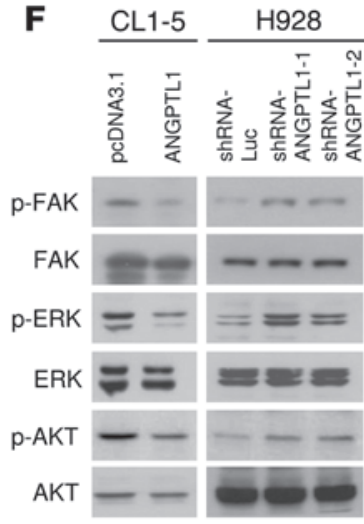

\section{Figure 5}

ANGPTL1 interacts with integrin $\alpha_{1} \beta_{1}$ and inhibits the downstream FAK/ERK pathway. (A) CL1-5 cells were treated with ANGPTL1 CM for 2 hours. Cell lysates were immunoprecipitated with anti-V5 and normal IgG. Western blot analysis was of V5 and integrin $\alpha_{1},-\alpha_{2},-\alpha_{3},-\alpha_{5},-\alpha_{6},-\alpha_{V},-\alpha_{8}$, $-\beta_{1},-\beta_{3},-\beta_{4}$, and $-\beta_{5}$ in this immunoprecipitation. The arrowhead indicates IgG. (B) Dose-dependent ANGPTL1 binding to immobilized integrin $\alpha_{1} \beta_{1}$ (top) and relative binding of collagen I, ANGPTL1, ANGPTL1, and indicated antibodies and BSA (bottom) with immobilized integrin $\alpha_{1} \beta_{1}$ by ELISA. (C) Surface plasmon resonance assay shows the binding profiles between immobilized integrin $\alpha_{1} \beta_{1}$ and ANGPTL1. Sensorgram was corrected against a reference flow cell with no immobilized protein. $K_{\mathrm{D}}=6.35 \times 10^{-8} \mathrm{M}$ was determined after global fitting (Langmuir 1:1 model) using Scrubber2. (D) Western blot analysis of activated integrin $\beta_{1}$ in ANGPTL1-overexpressing CL1-5 cells. (E) Western blot analysis of phosphorylation of FAK, ERK, AKT, JNK, and p38 in CL1-5 cells treated with $50 \mathrm{ng} / \mathrm{ml}$ rANGPTL1 for various periods of time. (F) Western blot analysis of phosphorylation of FAK, ERK, and AKT in ANGPTL1-overexpressing CL1-5 cells and ANGPTL1 knockdown H928 cells.

cancers (43-47). Furthermore, SLUG acts as a predictive marker for the clinical outcome of patients with lung adenocarcinoma, breast, ovarian, colon, and gastric cancers $(43,48-50)$. Recent reports have shown that SLUG promotes resistance to EGFR tyrosine kinase inhibitor target therapy (51) and that its expression in lung adenocarcinoma and breast cancer cells is associated with cancer stem cell properties (52-54). Both drug resistance and cancer stem cell features have the tendency to have tumorigenic or metastatic capa- bilities. In our results, we found that ANGPTL1 induces the MET process through posttranscriptional downregulation of SLUG. Therefore, we suggest that ANGPTL1 may be a potential target for developing new therapies for managing cancer metastasis.

The miR-200 family has been shown to play an important role in tumor progression through the targeting of the E-cadherin transcriptional repressors ZEB1 and ZEB2 (15, 25-27). A recent report has shown that miR-124a and miR-203 play critical roles in 
A
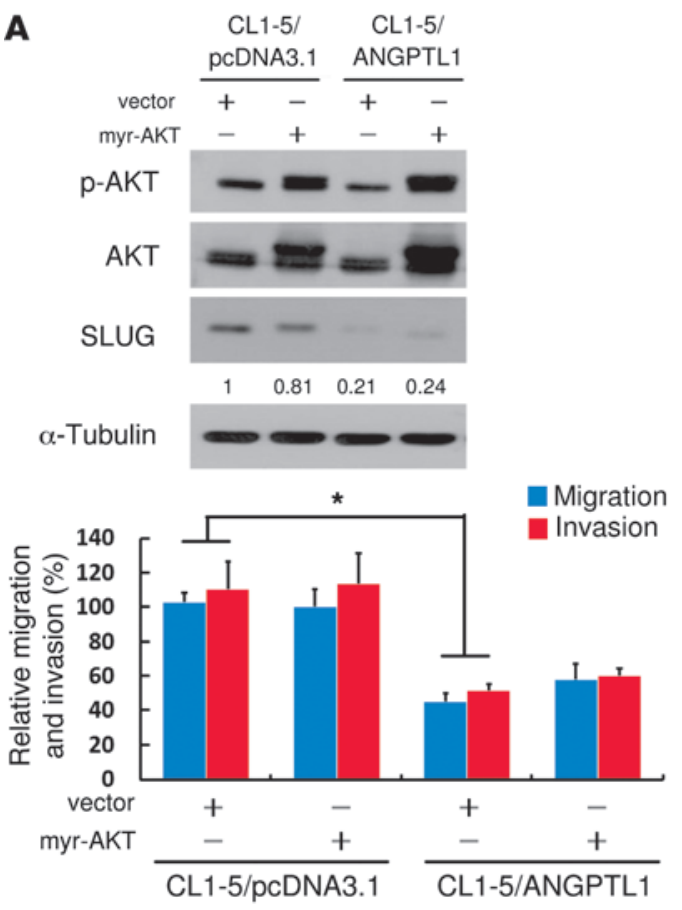

C

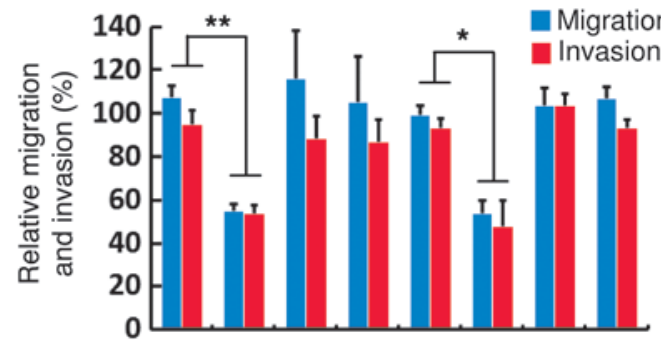

rANGPTL1 $(50 \mathrm{ng} / \mathrm{ml})$

CL1-5:
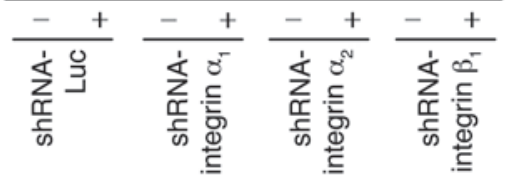

E

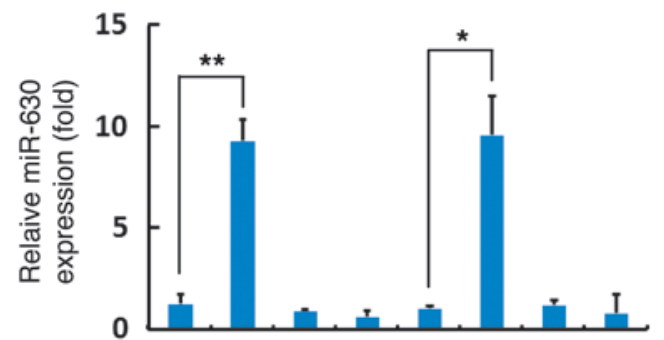

rANGPTL1 $(50 \mathrm{ng} / \mathrm{ml})$

CL1-5:

B
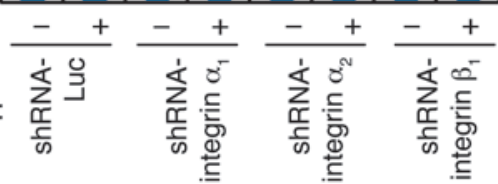
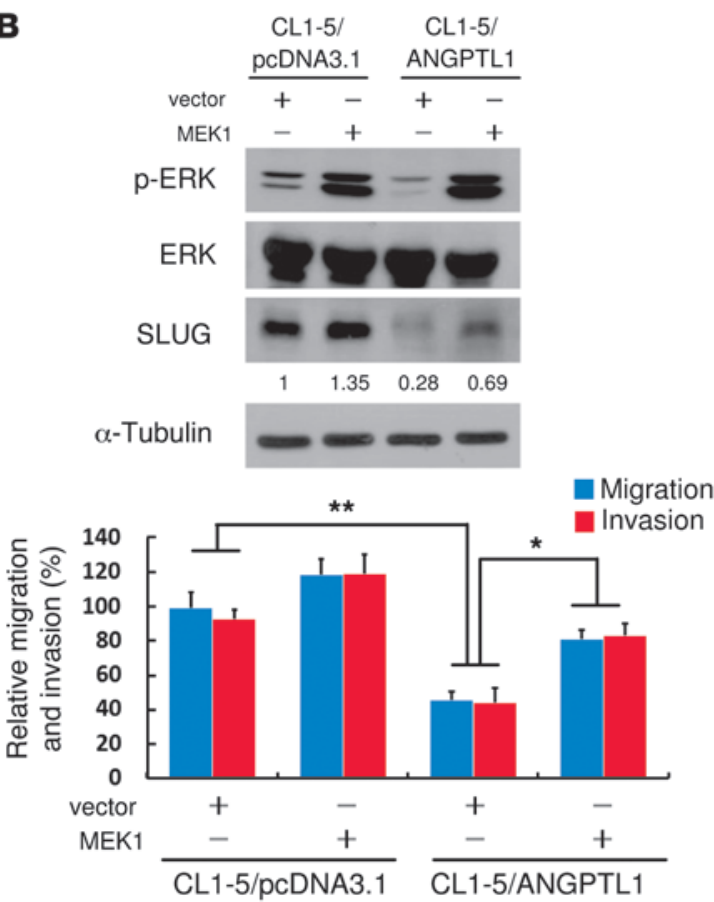

D CL1-5: ShRNA- ShRNA- ShRNA- ShRNA$\underline{\text { Luc }}$ integrin $\alpha_{1}$ integrin $\alpha_{2}$ integrin $\beta_{1}$ rANGPTL1 $(50 \mathrm{ng} / \mathrm{ml})$ SLUG $\alpha$-Tubulin
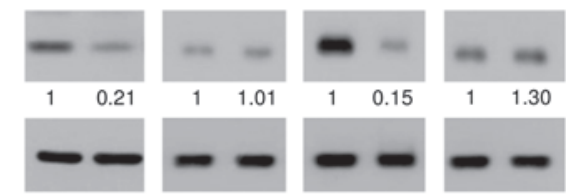

$\mathbf{F}$

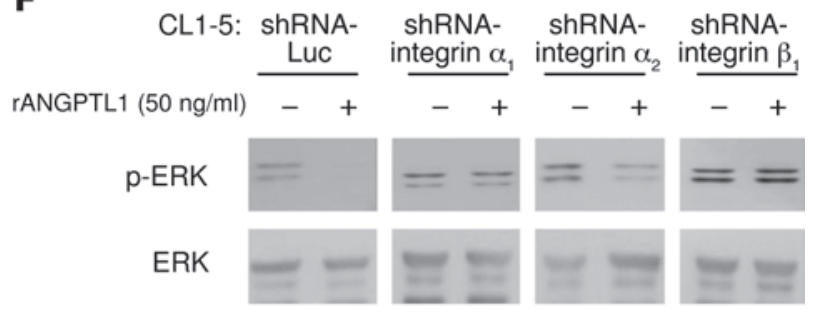

Figure 6

ANGPTL1 represses SLUG expression through the integrin $\alpha_{1} \beta_{1} / E R K / m i R-630$ pathway. (A and $\mathbf{B}$ ) Western blot analysis of phosphorylation of AKT, ERK, and SLUG and measurement of the migration and invasion in ANGPTL1-overexpressing CL1-5 cells transiently transfected with (A) myr-AKT- and (B) MEK1-expressing vectors. Numbers above the blots represent SLUG levels. (C) Measurement of the migration and invasion of CL1-5 cells infected with shRNA-Luc, shRNA-integrin $\alpha_{1}$, shRNA-integrin $\alpha_{2}$, and shRNA-integrin $\beta_{1}$ after treating with $50 \mathrm{ng} / \mathrm{ml}$ rANGPTL1. (D) Western blot analysis of SLUG in CL1-5 cells infected with shRNA-Luc, shRNA-integrin $\alpha_{1}$, shRNA-integrin $\alpha_{2}$, and shRNA-integrin $\beta_{1}$ by treating with $50 \mathrm{ng} / \mathrm{ml}$ rANGPTL1. (E) qRT-PCR analysis of miR-630 expression in CL1-5 cells infected with shRNA-Luc, shRNA-integrin $\alpha_{1}$, shRNAintegrin $\alpha_{2}$, and shRNA-integrin $\beta_{1}$ by treating with $50 \mathrm{ng} / \mathrm{ml}$ rANGPTL1. (F) Western blot analysis of phosphorylation of ERK in CL1-5 cells infected with shRNA-Luc, shRNA-integrin $\alpha_{1}$, shRNA-integrin $\alpha_{2}$, and shRNA-integrin $\beta_{1}$ by treating with 50 ng/ml rANGPTL1. Data are shown as mean \pm SEM. ${ }^{*} P<0.05,{ }^{* *} P<0.01$ (2-tailed Student's $t$ test). 
A

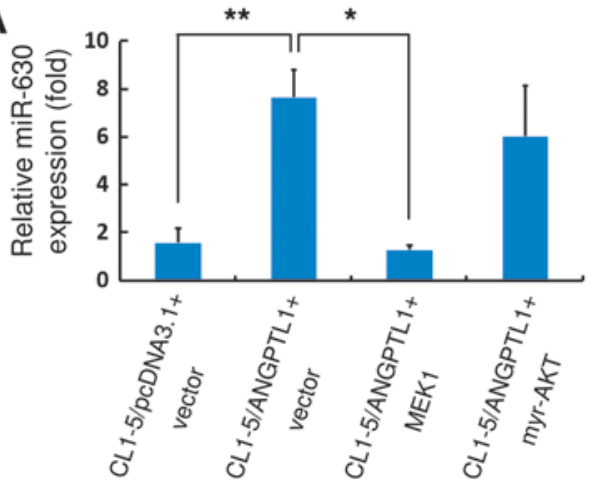

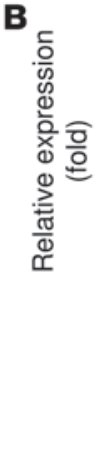

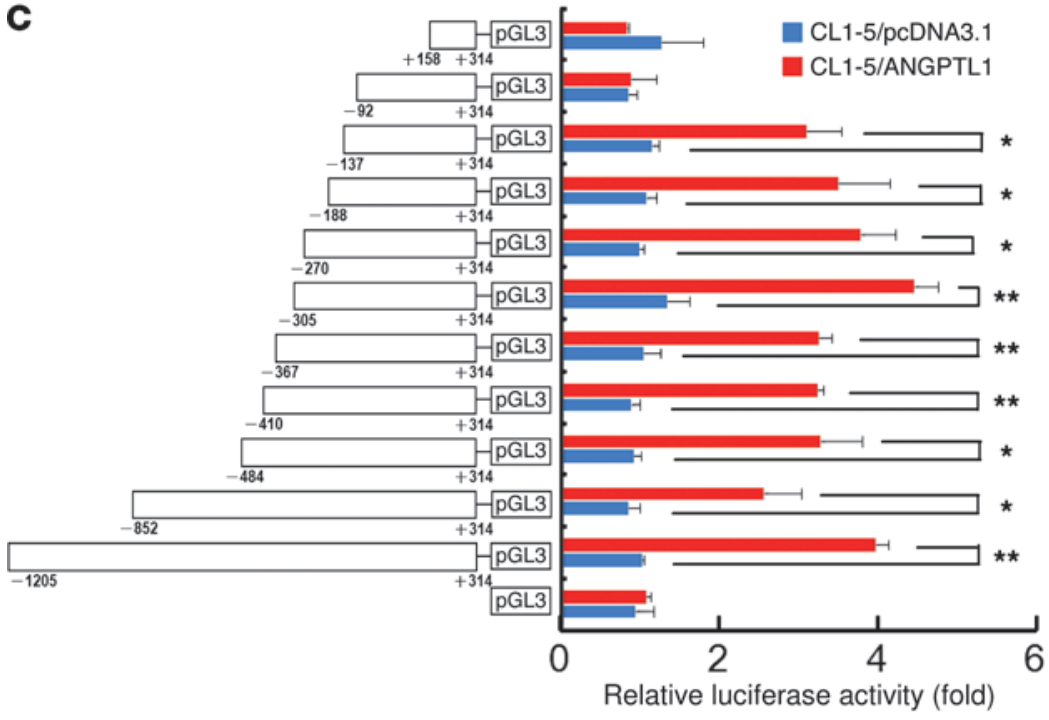

D $\operatorname{miR}-630$ promoter

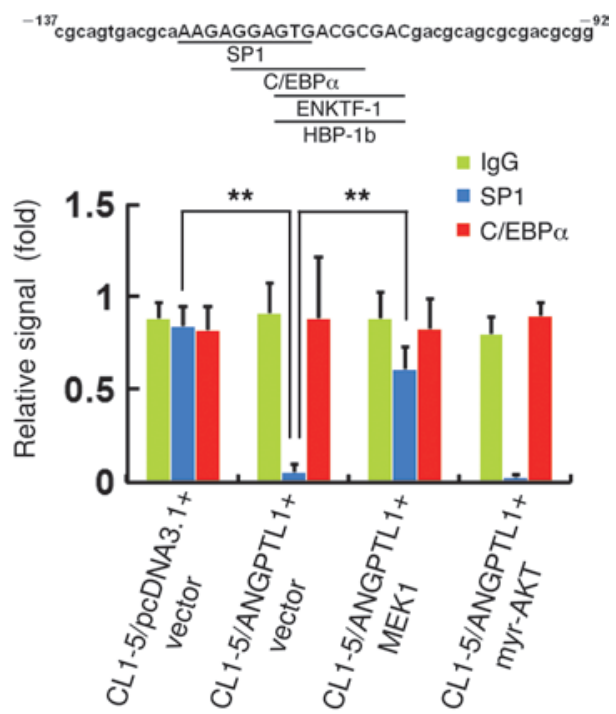

E

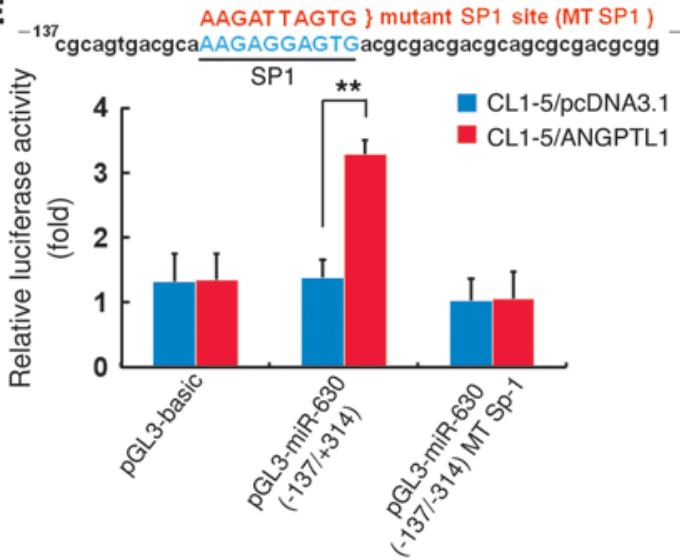

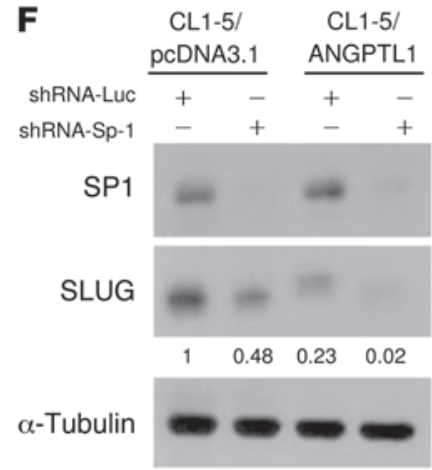

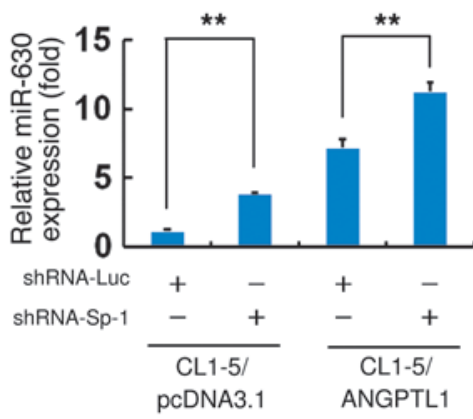

Figure 7

ANGPTL1 induces miR-630 transcription through repression of the ERK/SP1 pathway. (A) qRT-PCR analysis of miR-630 expression in ANGPTL1-overexpressing CL1-5 cells transiently transfected with myr-AKT and MEK1. (B) qRT-PCR analysis of pre-miR-630 and pri-miR-630 expression in ANGPTL1-overexpressing CL1-5 cells transiently transfected with myr-AKT and MEK1. (C) Schematic representation of various miR-630 promoter reporters (left). CL1-5/pcDNA3.1 and CL1-5/ANGPTL1 cells were transfected with various miR-630 promoter reporters or the pGL3-basic vector, and the luciferase activity was measured (right). (D) Schematic representation of the 46-bp region of human miR-630 promoter (-137 to -92 bp), where underlining indicates potential binding sites (top). Quantitative ChIP (qChIP) analysis of the miR-630 promoter region in ANGPTL1-overexpressing CL1-5 cells transiently transfected with myr-AKT and MEK1 (bottom). Signal is relative to the CL1-5/pcDNA3.1 plus vector group. (E) Schematic representation of the 46-bp region of human miR-630 promoter ( -137 to $-92 \mathrm{bp}$ ), where underlining indicates the SP1-binding site and mutated SP1-binding site (top). CL1-5/pcDNA3.1 and CL1-5/ANGPTL1 cells were transfected with miR-630 promoter reporter ( -137 to -92 bp), miR-630 promoter reporter ( -137 to -92 bp) SP1 mutant construct (MT SP1), or the pGL3-basic vector, and the luciferase activity was measured (bottom). (F) Western blot analysis of SP1 and SLUG, and qRT-PCR analysis of miR-630 expression in ANGPTL1-overexpressing CL1-5 cells infected with shRNA-Luc and shRNA-SP1. Data are shown as mean \pm SEM. ${ }^{*} P<0.05,{ }^{* *} P<0.01$ (2-tailed Student's $t$ test). 
inhibiting human embryonic stem cells from differentiating into embryoid bodies and preventing invasiveness, respectively, by targeting SLUG $(55,56)$. However, our miRNA microarray data indicated that miR-124a and miR-203 were not significantly altered after ANGPTL1 overexpression. The expression of miRNAs often exhibits developmental stage-specific or tissue-specific patterns, and their regulation occurs transcriptionally or posttranscriptionally $(16,17)$. Here, we elucidate a mechanism whereby ANGPTL1 represses lung cancer cell motility and invasiveness through downregulation of SLUG by inducing miR-630 transcription.

Numerous studies have shown that ANGPTLs transduce their signals via integrin pathways. ANGPTL3 induces the adhesion and migration of endothelial cells via integrin $\alpha_{v} \beta_{3}$ (29). ANGPTL4 interacts with integrin $\beta_{3}$ and integrin $\beta_{5}$ to escape anoikis in tumors and control the migration of keratinocytes $(30,33)$. Activation of integrin downstream signaling, such as FAK and ERK phosphorylation, strongly correlates with migration and invasion of cancer cells $(57,58)$. Our results showed that ANGPTL1 represses the integrin/FAK/ERK pathway and knockdown of a specific collagen-associated integrin, integrin $\alpha_{1} \beta_{1}$, attenuates ANGPTL1-regulated signaling and subsequent cancer cell behaviors. In addition, in our results showed that ANGPTL1 also could reduce cell adhesion to fibronectin and vitronectin. Therefore, ANGPTL1 may interact with other integrins to execute other cell functions. The details of the adhesion inhibition mechanism will be studied in the future.

In summary, we provide clinical evidence that ANGPTL1 expression inversely correlates with advanced-stage lymph node metastasis and positively correlates with survival of patients with cancer. We demonstrate that ANGPTL1 represses lung cancer cell migration and invasion by regulating the integrin $\alpha_{1} \beta_{1} /$ FAK/ERK/SP1/ miR-630/SLUG signaling axis.

\section{Methods}

Cell culture, plasmids, transfection, and recombinant protein. Lung adenocarcinoma cell lines (CL1-0 and CL1-5) were established at the National Health Research Institutes laboratory and displayed progressively increasing invasiveness (21). Lung cancer cell lines (CL1-0, CL1-5, PC14, PC9, H928, H1299, and A549) were grown in RPMI-1640 medium supplemented with $10 \%$ FBS. 293T cells were grown in DMEM medium supplemented with $10 \%$ FBS. The MDA-MB-231/I3 subline was selected from parental MDA-MB-231 cells as CL1-0 and CL1-5. Breast cancer cell lines (MCF-7, MDA-MB-231, and MDA-MB-231/I3) were grown in DMEM/F12 medium supplemented with $10 \% \mathrm{FBS}$. All cell lines were grown at $37^{\circ} \mathrm{C}$ in a humidified 5\% $\mathrm{CO}_{2}$ atmosphere. Full-length ANGPTL1, ANGPTL1-CCD, and ANGPTL1-FD plasmids were cloned into the pcDNA3.1/V5-His Topo vector (Invitrogen). pCIneo-SLUG and PLKO_AS.neo/SLUG were a gift from Yang Pan-Chyr (Department of Internal Medicine, National Taiwan University Hospital). The constitutively activated MEK1 plasmid was provided by Ruey-Hwa Chen (Institute of Biological Chemistry, Academia Sinica). The myr-Akt plasmid was provided by Ching-Chow Chen (Department of Pharmacology, National Taiwan University). miRNA constructs expressing miR-545 and miR-630 were designed by our laboratory, and DNA was synthesized by a biotech company (Mission Biotech). In brief, the pre-miRNA was ligated into a BLOCK-iT Pol II miR RNAi expression vector. Transfections were carried out using Lipofectamine 2000 (Invitrogen) according to the manufacturer's instructions. Established stable cell lines were generated by transfection with Lipofectamine 2000 followed by selection with G418 (500 $\mu \mathrm{g} / \mathrm{ml})$. rANGPTL1 was purchased from Abnova. Integrin $\alpha_{1} \beta_{1}$ recombinant protein was purchased from R\&D Systems.
Protein stability assay and Western blot analysis. For protein stability assays, cells were incubated with cycloheximide $(10 \mu \mathrm{g} / \mathrm{ml}$, Sigma-Aldrich) to inhibit further protein synthesis and incubated with MG132 $(10 \mu \mathrm{M}$, Sigma-Aldrich) to inhibit $26 \mathrm{~S}$ proteasome; cells were immediately harvested. Proteins in the total cell lysates were separated on $10 \%-12 \%$ SDSPAGE and electrotransferred to a polyvinylidene difluoride membrane (Millipore). After the blots were blocked in a solution of $10 \%$ skimmed milk, $0.1 \%$ Tween 20 , and PBS, membrane-bound proteins were probed with primary antibodies (primers used are shown in Supplemental Table 1; antibodies used are shown in Supplemental Table 2) overnight $4^{\circ} \mathrm{C}$. The membranes were washed and then incubated with horseradish peroxidaseconjugated secondary antibodies for 45 minutes. Antibody-bound protein bands were detected using enhanced chemiluminescence reagents (Millipore) and photographed with Kodak X-Omat Blue autoradiography film (Perkin Elmer Life Sciences).

Immunoprecipitation. CL1-5 cells were incubated in ANGPTL1 CM at $37^{\circ} \mathrm{C}$ for 2 hours. Cells were lysed by brief sonication in coimmunoprecipitation buffer (20 mM Tris- $\mathrm{HCl}$ [pH 7.4], $150 \mathrm{mM} \mathrm{NaCl}, 1 \mathrm{mM} \mathrm{CaCl}_{2}$, $2 \mathrm{mM} \mathrm{MnCl}_{2}, 1.2 \%$ Triton X-100) supplemented with protease inhibitors cocktails (Sigma-Aldrich) as previously described (59). Lysates were centrifuged for 30 minutes at $14,500 \mathrm{~g}$, and the resulting supernatant was precleared by incubation with immobilized Protein A/G gel ( $20 \mu \mathrm{l}$; Pierce) for 1 hour at $4^{\circ} \mathrm{C}$. The precleared supernatant was subjected to overnight immunoprecipitation using the indicated antibodies (Supplemental Table 2) or control IgG antibodies at $4^{\circ} \mathrm{C}$. The next day, protein complexes were collected by incubation with $35 \mu \mathrm{l}$ of immobilized Protein A/G gel for 1 hour at $4^{\circ} \mathrm{C}$. The collected protein complexes were washed 5 times with coimmunoprecipitation buffer and eluted by boiling in protein sample buffer under reducing conditions, after which proteins were resolved by SDS-PAGE and analyzed by Western blot.

Surface plasmon resonance. Integrin $\alpha_{1} \beta_{1}$ recombinant protein was immobilized onto the ProteOn GLC chip by amine coupling, according to the manufacturer's instructions (Bio-Rad). Different concentrations of ANGPTL1 were introduced into the GLC chip at a flow rate of $30 \mu \mathrm{l} / \mathrm{min}$ for 5 minutes with running buffer (1X PBS, pH 7.2). Global fitting of the data to a Langmuir 1:1 model was used to determine the association $\left(k_{\mathrm{a}}\right)$, dissociation $\left(k_{\mathrm{d}}\right)$, and affinity constants $\left(K_{\mathrm{D}}\right)$ using Scrubber2 (BioLogic Software).

Animal studies. All animal experiments were done in accordance with a protocol approved by the National Taiwan University College of Medicine and National Taiwan University College of Public Health Institutional Animal Care and Use Committees. Female SCID mice (6-8 weeks old) were used. For experimental metastasis assays, $1 \times 10^{6}$ cells were resuspended in $0.1 \mathrm{ml}$ PBS and injected into the lateral tail vein. Lung metastatic progression or nodules were monitored and quantified either using the noninvasive bioluminescence system (IVIS-Spectrum) or by counting under dissecting microscope. For orthotopic metastasis assays, $5 \times 10^{5}$ (CL1-5) cells and $1 \times 10^{6}(\mathrm{CL} 1-0)$ cells were resuspended in a 1:1 mixture of PBS and GFR-Matrigel (BD). This mixture was then injected into the left lateral thorax of each mouse, as previously described (60). The metastatic nodules in the right lung were quantified using a dissecting microscope.

miRNA microarray and computational analysis. Total RNA from CL1-5/ pcDNA3.1 and CL1-5/ANGPTL1 cells was isolated. The expression of 365 mature human miRNAs in the cells was profiled using real-time PCR (MicroRNA TaqMan Low Density Array Analysis, Applied Biosystems) and was normalized to RNU48. Microarray data have been deposited in the NCBI GEO database, with accession number GSE42481. We used 3 online computational algorithms (TargetScan 5.0-5.2 version, PicTar, and miRanda 2008), which predicted the binding potential of miRNAs to the $3^{\prime}$-UTR region of SNAI2 and filtered out 10 candidate miRNAs. 
Statistics. The data are presented as the mean \pm SEM. The 2-tailed Student's $t$ test was used to compare data between 2 groups. Statistical analyses of clinicopathological data were performed using Pearson's $\chi^{2}$ test. Survival curves were analyzed using the Kaplan-Meier method and log-rank test. $P$ values of less than 0.05 were considered to be statistically significant.

Study approval. Tissue preparation and analysis were approved by the Institutional Review Board of National Taiwan University Hospital, and patients provided informed consent. Animal studies were approved by Institutional Animal Care and Use Committees of National Taiwan University College of Medicine and College of Public Health.

\section{Acknowledgments}

This work was supported by the National Science Council grant from Taiwan (NSC 101-2320-B-039-044-MY3, NSC99-2314-B039-002-MY3, NSC 99-2632-B-039-001-MY3 to J.-L. Su); National Health Research Institutes grant from Taiwan (NHRI-EX1009712BC to J.-L. Su); Department of Health, Executive Yuan grant from Taiwan (DOH 101-TD-C-111-005, DOH 101-TD-PB-111NSC015 to J.-L. Su); National Science Council grant from Taiwan
(NSC 101-2320-B-006-045-MY2 to P.-S. Chen); grants from China Medical University (CMU101-NSC-04 to J.-L. Su) and National Taiwan University Cutting-Edge Steering Research Project (101R7601-1 to M.-L. Kuo); and National Science Council grant from Taiwan (NSC 101-2320-B-002-042, NSC 101-2911-I-002-303 to M.-L. Kuo).

Received for publication March 29, 2012, and accepted in revised form December 19, 2012.

Address correspondence to: Min-Liang Kuo, Graduate Institute of Biochemical Sciences, College of Life Science, National Taiwan University, No. 1, Section 4, Roosevelt Road, Taipei 106, Taiwan. Phone: 886.2.2312.3456.88607; Fax: 886.2.2341.0217; E-mail: kuominliang@ntu.edu.tw (M.-L. Kuo). Or to: Jen-Liang $\mathrm{Su}$, Graduate Institute of Cancer Biology, College of Medicine, China Medical University, Taichung 40402, Taiwan. Phone: 886.4.2205.2121.7932; Fax: 886.4.2207.9142; E-mail: jlsu@mail. cmu.edu.tw (J.-L. Su).
1. Chambers AF, Groom AC, MacDonald IC. Dissemination and growth of cancer cells in metastatic sites. Nat Rev Cancer. 2002;2(8):563-572.

2. Fidler IJ. The pathogenesis of cancer metastasis: the 'seed and soil' hypothesis revisited. Nat Rev Cancer. 2003;3(6):453-458.

3. Hato T, Tabata M, Oike Y. The role of angiopoietin-like proteins in angiogenesis and metabolism. Trends Cardiovasc Med. 2008;18(1):6-14.

4. Galaup A, et al. Angiopoietin-like 4 prevents metastasis through inhibition of vascular permeability and tumor cell motility and invasiveness. Proc Natl Acad Sci U S A. 2006;103(49):18721-18726.

5. Oike Y, Akao M, Kubota Y, Suda T. Angiopoietin-like proteins: potential new targets for metabolic syndrome therapy. Trends Mol Med. 2005;11(10):473-479.

6. Kersten S. Regulation of lipid metabolism via angiopoietin-like proteins. Biochem Soc Trans. 2005;33(5):1059-1062.

7. Zhang CC, et al. Angiopoietin-like proteins stimulate ex vivo expansion of hematopoietic stem cells Nat Med. 2006;12(2):240-245.

8. Tabata M, et al. Angiopoietin-like protein 2 promotes chronic adipose tissue inflammation and obesity-related systemic insulin resistance. Cell Metab. 2009;10(3):178-188.

9. Smagur A, Szary J, Szala S. Recombinant angioarrestin secreted from mouse melanoma cells inhibits growth of primary tumours. Acta Biochim Pol. 2005;52(4):875-879.

10. Dhanabal M, Jeffers M, LaRochelle WJ, Lichenstein HS. Angioarrestin: a unique angiopoietin-related protein with anti-angiogenic properties. Biochem Biophys Res Commun. 2005;333(2):308-315.

11. Polyak K, Weinberg RA. Transitions between epithelial and mesenchymal states: acquisition of malignant and stem cell traits. Nat Rev Cancer. 2009;9(4):265-273.

12. Yang J, et al. Twist, a master regulator of morphogenesis, plays an essential role in tumor metastasis. Cell. 2004;117(7):927-939.

13. Perez-Mancera PA, et al. SLUG in cancer development. Oncogene. 2005;24(19):3073-3082.

14. Carver EA, Jiang R, Lan Y, Oram KF, Gridley T. The mouse snail gene encodes a key regulator of the epithelial-mesenchymal transition. Mol Cell Biol. 2001;21(23):8184-8188.

15. Park SM, Gaur AB, Lengyel E, Peter ME. The miR200 family determines the epithelial phenotype of cancer cells by targeting the E-cadherin repressors ZEB1 and ZEB2. Genes Dev. 2008;22(7):894-907.
16. Ambros $\mathrm{V}$. The functions of animal microRNAs. Nature. 2004;431(7006):350-355.

17. Bartel DP. MicroRNAs: genomics, biogenesis, mechanism, and function. Cell. 2004;116(2):281-297.

18. Esquela-Kerscher A, Slack FJ. Oncomirs microRNAs with a role in cancer. Nat Rev Cancer. 2006;6(4):259-269.

19. Yu YP, et al. Gene expression alterations in prostate cancer predicting tumor aggression and preceding development of malignancy. J Clin Oncol. 2004;22(14):2790-2799.

20. Lapointe J, et al. Gene expression profiling identifies clinically relevant subtypes of prostate cancer. Proc Natl Acad Sci U S A. 2004;101(3):811-816.

21. Chu YW, et al. Selection of invasive and metastatic subpopulations from a human lung adenocarcinoma cell line. Am J Respir Cell Mol Biol. 1997;17(3):353-360.

22. Wang SP, et al. p53 controls cancer cell invasion by inducing the MDM2-mediated degradation of Slug. Nat Cell Biol. 2009;11(6):694-704.

23. Martin TA, Goyal A, Watkins G, Jiang WG. Expression of the transcription factors snail, slug, and twist and their clinical significance in human breast cancer. Ann Surg Oncol. 2005;12(6):488-496.

24. Uchikado Y, et al. Slug Expression in the E-cadherin preserved tumors is related to prognosis in patients with esophageal squamous cell carcinoma. Clin Cancer Res. 2005;11(3):1174-1180.

25. Korpal M, Lee ES, Hu G, Kang Y. The miR-200 family inhibits epithelial-mesenchymal transition and cancer cell migration by direct targeting of E-cadherin transcriptional repressors ZEB1 and ZEB2. J Biol Chem. 2008;283(22):14910-14914.

26. Korpal M, Kang Y. The emerging role of miR-200 family of microRNAs in epithelial-mesenchymal transition and cancer metastasis. RNA Biol. 2008;5(3):115-119.

27. Burk $U$, et al. A reciprocal repression between ZEB1 and members of the miR-200 family promotes EMT and invasion in cancer cells. EMBO Rep. 2008;9(6):582-589

28. Ma L, et al. miR-9, a MYC/MYCN-activated microRNA, regulates E-cadherin and cancer metastasis. Nat Cell Biol. 2010;12(3):247-256.

29. Li Y, et al. Angiopoietin-like protein 3 modulates barrier properties of human glomerular endothelial cells through a possible signaling pathway involving phosphatidylinositol-3 kinase/protein kinase B and integrin alphaVbeta3. Acta Biochim Biophys Sin (Shanghai). 2008; 40(6):459-465.
30. Zhu P, et al. Angiopoietin-like 4 protein elevates the prosurvival intracellular $\mathrm{O} 2(-): \mathrm{H} 2 \mathrm{O} 2$ ratio and confers anoikis resistance to tumors. Cancer Cell. 2011;19(3):401-415.

31. Camenisch G, et al. ANGPTL3 stimulates endothelial cell adhesion and migration via integrin alpha vbeta 3 and induces blood vessel formation in vivo. J Biol Chem. 2002;277(19):17281-17290.

32. Huang RL, et al. ANGPTL4 modulates vascular junction integrity by integrin signaling and disruption of intercellular VE-cadherin and claudin-5 clusters. Blood. 2011;118(14):3990-4002.

33. Goh YY, et al. Angiopoietin-like 4 interacts with integrins beta 1 and beta5 to modulate keratinocyte migration. Am J Pathol. 2010;177(6):2791-2803.

34. Luque A, Gomez M, Puzon W, Takada Y, Sanchez-Madrid F, Cabanas C. Activated conformations of very late activation integrins detected by a group of antibodies (HUTS) specific for a novel regulatory region (355-425) of the common beta 1 chain. J Biol Chem. 1996;271(19):11067-11075.

35. Yang C, Zeisberg M, Lively JC, Nyberg P, Afdhal N, Kalluri R. Integrin alpha1beta 1 and alpha2beta 1 are the key regulators of hepatocarcinoma cell invasion across the fibrotic matrix microenvironment. Cancer Res. 2003;63(23):8312-8317.

36. Baronas-Lowell D, et al. Differential modulation of human melanoma cell metalloproteinase expression by alpha2beta 1 integrin and CD 44 triple-helical ligands derived from type IV collagen. J Biol Chem. 2004;279(42):43503-43513.

37. Elshaw SR, et al. A comparison of ocular melanocyte and uveal melanoma cell invasion and the implication of alpha1beta1, alpha4beta 1 and alpha6beta1 integrins. Br J Ophthalmol. 2001; 85(6):732-738.

38. Ibaragi $S$, et al. Induction of MMP-13 expression in bone-metastasizing cancer cells by type I collagen through integrin alpha1beta 1 and alpha2beta1-p38 MAPK signaling. Anticancer Res. 2011;31(4):1307-1313.

39. Vuoristo M, Vihinen P, Vlaykova T, Nylund C, Heino J, Pyrhonen S. Increased gene expression levels of collagen receptor integrins are associated with decreased survival parameters in patients with advanced melanoma. Melanoma Res. 2007;17(4):215-223.

40. Jack GD, Zhang L, Friedman AD. M-CSF elevates c-Fos and phospho-C/EBPalpha(S21) via ERK whereas G-CSF stimulates SHP2 phosphorylation in marrow progenitors to contribute to myeloid lineage specification. Blood. 2009;114(10):2172-2180. 
41. Tan NY, Khachigian LM. Sp1 phosphorylation and its regulation of gene transcription. Mol Cell Biol. 2009;29(10):2483-2488.

42. Dhanabal M, et al. Angioarrestin: an antiangiogenic protein with tumor-inhibiting properties. Cancer Res. 2002;62(13):3834-3841.

43. Shih JY, et al. Transcription repressor slug promotes carcinoma invasion and predicts outcome of patients with lung adenocarcinoma. Clin Cancer Res. 2005;11(22):8070-8078.

44. Kurrey NK, K A, Bapat SA. Snail and Slug are major determinants of ovarian cancer invasiveness at the transcription level. Gynecol Oncol. 2005;97(1):155-165.

45. Chen M, Chen LM, Chai KX. Androgen regulation of prostasin gene expression is mediated by sterol-regulatory element-binding proteins and SLUG. Prostate. 2006;66(9):911-920.

46. Zhang K, et al. Slug enhances invasion ability of pancreatic cancer cells through upregulation of matrix metalloproteinase- 9 and actin cytoskeleton remodeling. Lab Invest. 2011;91(3):426-438.

47. Yang HW, Menon LG, Black PM, Carroll RS, Johnson MD. SNAI2/Slug promotes growth and invasion in human gliomas. BMC Cancer. 2010;10:301.

48. Elloul S, et al. Snail, Slug, and Smad-interacting protein 1 as novel parameters of disease aggressive- ness in metastatic ovarian and breast carcinoma. Cancer. 2005;103(8):1631-1643.

49. Shioiri M, et al. Slug expression is an independent prognostic parameter for poor survival in colorectal carcinoma patients. $\mathrm{Br} J$ Cancer. 2006;94(12):1816-1822.

50. Uchikado Y, et al. Increased Slug and decreased E-cadherin expression is related to poor prognosis in patients with gastric cancer. Gastric Cancer. 2011;14(1):41-49.

51. Chang TH, et al. Slug confers resistance to the epidermal growth factor receptor tyrosine kinase inhibitor. Am J Respir Crit Care Med. 2011; 183(8):1071-1079.

52. Chiou SH, et al. Coexpression of Oct4 and Nanog enhances malignancy in lung adenocarcinoma by inducing cancer stem cell-like properties and epithelial-mesenchymal transdifferentiation. Cancer Res. 2010;70(24):10433-10444.

53. Storci $G$, et al. TNFalpha up-regulates SLUG via the NF-kappaB/HIF1alpha axis, which imparts breast cancer cells with a stem cell-like phenotype. J Cell Physiol. 2010;225(3):682-691.

54. Bhat-Nakshatri P, et al. SLUG/SNAI2 and tumor necrosis factor generate breast cells with CD44+/ CD24- phenotype. BMC Cancer. 2010;10:411.
55. Lee MR, Kim JS, Kim KS. miR-124a is important for migratory cell fate transition during gastrulation of human embryonic stem cells. Stem Cells. 2010;28(9):1550-1559.

56. Zhang Z, Zhang B, Li W, Fu L, Zhu Z, Dong JT. Epigenetic silencing of miR-203 upregulates SNAI2 and contributes to the invasiveness of malignant breast cancer cells. Genes Cancer. 2011;2(8):782-791.

57. Mitra SK, Hanson DA, Schlaepfer DD. Focal adhesion kinase: in command and control of cell motility. Nat Rev Mol Cell Biol. 2005;6(1):56-68.

58. Liao YC, Shih YW, Chao CH, Lee XY, Chiang TA. Involvement of the ERK signaling pathway in fisetin reduces invasion and migration in the human lung cancer cell line A549. J Agric Food Chem. 2009;57(19):8933-8941.

59. Hu B, Jarzynka MJ, Guo P, Imanishi Y, Schlaepfer DD, Cheng SY. Angiopoietin 2 induces glioma cell invasion by stimulating matrix metalloprotease 2 expression through the alphavbeta 1 integrin and focal adhesion kinase signaling pathway. Cancer Res. 2006;66(2):775-783

60. Onn A, et al. Development of an orthotopic model to study the biology and therapy of primary human lung cancer in nude mice. Clin Cancer Res. 2003;9(15):5532-5539. 